\title{
Mathematical Simulation of the lonospheric Electric Field as a Part of the Global Electric Circuit
}

\author{
V. V. Denisenko ${ }^{1,2} \cdot$ M. J. Rycroft ${ }^{3,4} \cdot$ R. G. Harrison ${ }^{5,6}$
}

Received: 16 April 2018 / Accepted: 1 September 2018 / Published online: 15 September 2018

(c) The Author(s) 2018

\begin{abstract}
Electric currents flowing in the global electric circuit are closed by ionospheric currents. A model for the distribution of the ionospheric potential which drives these currents is constructed. Only the internal electric fields and currents generated by thunderstorms are studied, and without any magnetospheric current sources or generators. The atmospheric conductivity profiles with altitude are empirically determined, and the topography of the Earth's surface is taken into account. A two-dimensional approximation of the ionospheric conductor is based on high conductivities along the geomagnetic field; the Pedersen and Hall conductivity distributions are calculated using empirical models. The values of the potential in the E- and F-layers of the ionosphere are not varied along a magnetic field line in such a model and the electric field strength is only slightly varied because the segments of neighboring magnetic field lines are not strictly parallel. It is shown that the longitudinal and latitudinal components of the ionospheric electric field of the global electric circuit under typical conditions for July, under high solar activity, at the considered point in time, 19:00 UT, do not exceed $9 \mu \mathrm{V} / \mathrm{m}$, and in the sunlit ionosphere they are less than $2 \mu \mathrm{V} / \mathrm{m}$. The calculated maximum potential difference in the E- and F-layers is $42 \mathrm{~V}$; the maximum of the potential occurs above African thunderstorms that are near the terminator at that time. A weak local maximum also exists above the thunderstorm area in Central America. The minimum potential occurs near midnight above the Himalayas. The potential has identical values at ionospheric conjugate points. The voltage increases to $55 \mathrm{~V}$ at 23:00 UT and up to $72 \mathrm{~V}$ at 06:00 UT, when local midnight comes, respectively, for the African and Central American thunderstorm areas. These voltages are about twice as large at solar minimum. With our more realistic ionospheric model, the electric fields are an order of magnitude smaller than those found in the well-known model of Roble and Hays ( $\mathrm{J}$ Geophys Res 84(A12):7247-7256, 1979). Our simulations quantitatively support the traditional presentation of the ionosphere as an ideal conductor in models of the global electric circuit, so that our model can be used to investigate UT variations of the global electric circuit.
\end{abstract}

Keywords Global electric circuit · Ionosphere · Electric field · Mathematical simulation

V. V. Denisenko

denisen@icm.krasn.ru

Extended author information available on the last page of the article 


\section{Introduction}

The main interest in the ionosphere is due to its influence on the propagation of radio waves due to a change of the refractive index of the medium from the value of 1 . The real part of the refractive index is determined by the concentration of electrons, and the imaginary part depends also on electron collision frequencies, which, in turn, depend on the concentrations and temperatures of other charged and neutral particles. The formation of space distributions of all these parameters is essentially affected by the electric field, since it determines the drift of charged particles in the geomagnetic field.

There are several mechanisms of ionospheric electric field generation. First of all, there are magnetohydrodynamic processes in the magnetosphere, associated with the interaction of the solar wind with the Earth's magnetic dipole. These phenomena create currents from the magnetosphere into the high-latitude regions of the ionosphere whose strength may be several millions of amperes (Hargreaves 1979). Closure of these currents in the ionosphere occurs due to electric fields with a strength of up to $100 \mathrm{mV} / \mathrm{m}$. These fields extend to the entire ionosphere; a field of $\mathrm{mV} / \mathrm{m}$ order of magnitude penetrates even up to the geomagnetic equator (Denisenko and Zamay 1992).

The second most important mechanism is winds in the upper atmosphere, which cause movement of the ionospheric medium. Due to this motion of the conducting medium in the geomagnetic field, the generation of electric fields with strength up to $10 \mathrm{mV} / \mathrm{m}$ occurs all over the globe (Hargreaves 1979).

There are also ionospheric electric fields due to the currents from the atmosphere. These are the currents of the global electric circuit (GEC), which are generated by thunderstorms. Although numerous articles analyze the GEC, its ionospheric part is still insufficiently studied. The present work is devoted to mathematical simulation of the ionospheric electric field of the GEC, more precisely, the part of the GEC generated by thunderstorms.

In accordance with the modern definition of the GEC (Mareev 2010), the ionospheric and atmospheric electric fields and currents generated in the magnetosphere are also included in the GEC. Such an approach is used in the model of Lucas et al. (2015), but the ionospheric electric fields generated by thunderstorms are not visible against the background of those generated in the magnetosphere. That is why we do not include these fields and currents into the actual model and study only a part of the GEC generated by thunderstorms.

Of course, there are other, relatively small-scale, generators of ionospheric electric fields. For example, perturbations of the electric field are observed in the atmosphere near ground before earthquakes. The analysis of the penetration of such fields into the ionosphere is presented in many papers, and considered in our article (Denisenko 2015). The maximum possible strength of the electric field penetrating the ionosphere associated with moderate earthquakes does not exceed $\mu \mathrm{V} / \mathrm{m}$ (Denisenko et al. 2013).

Modern views on the GEC are described, for example, in the review of Rycroft et al. (2008). In the last decade, there has been considerable progress in studies of different aspects of the GEC by many authors and groups, e.g., Aplin et al. (2008), Baumgaertner et al. (2013), Bayona et al. (2015), Jansky and Pasko (2014, 2015), Jansky et al. (2017), Kudintseva et al. (2016), Mareev and Volodin (2014), Odzimek et al. (2010), Rycroft et al. (2007, 2012), Rycroft and Harrison (2012), Slyunyaev et al. (2015), Tinsley (2008), Williams (2009) and Williams and Mareev (2014). The generator in the circuit is the set of all storm clouds occurring on the Earth. Thunderstorm clouds are mainly discharged by lightning, carrying charges to the land and other clouds, but there also exists a current flowing 
into the upper atmosphere and further into the ionosphere. The total current into the ionosphere is on average about one or two thousand amperes (Hays and Roble 1979).

This generator creates a potential difference of about $300 \mathrm{kV}$ between two good conductors: the ionosphere and the ground. Because of its large conductivity the upper atmosphere above $20 \mathrm{~km}$ has about the same potential as the ionosphere (Denisenko et al. 2009). This means that the simplified consideration of the ground-ionosphere as a spherical capacitor with the upper shell coinciding with the bottom of the conducting $\mathrm{D}$ region ionosphere is incorrect as is shown in Haldoupis et al. (2017). This effect is taken into account in our model because we use empirical models of the height distributions of the atmospheric conductivity.

Since the atmosphere is also a conductor, though a relatively poor one, charges return through it from the ionosphere to the ground. This vertical current of about $2 \mathrm{pA} / \mathrm{m}^{2}$ density is referred to as the fair weather current. It is associated with a vertical electric field, whose strength under fair weather conditions is about $130 \mathrm{~V} / \mathrm{m}$ near the ground. Since the currents from the thunderstorms penetrate into small regions of the ionosphere, and go down to ground from the whole ionosphere, there exists an ionospheric current to close the circuit. This current is generated by an electric field in the ionosphere, which is the subject of our study.

A great number of articles have described the GEC, and many detailed measurements have been made in the atmosphere. However, there is no measurement of the ionospheric part of this circuit because the currents involved are very small. A mathematical simulation helps in this situation. The model which we present here suggests what exactly should be found, or explains why observations are not possible.

The most detailed mathematical model of this field has been established for a long time in Hays and Roble (1979). Due to the limitations imposed by the mathematical methods used, its authors greatly simplified the distribution of the ionospheric conductivity. In accordance with Hargreaves (1979), the ionospheric current flowing across the magnetic field takes place at heights about $80-500 \mathrm{~km}$ and it can be approximately simulated with Pedersen and Hall conductivities integrated along magnetic field lines. In the model (Hays and Roble 1979), Hall conductivity was neglected and the integral Pedersen conductivity $\Sigma_{P}$ was taken as a constant. Focusing on the phenomenon at night they put $\Sigma_{P}=0.05 \mathrm{~S}$, while $\Sigma_{p}$ exceeds 10S in the sunlit ionosphere (Weimer 1999). The authors of the model (Hays and Roble 1979) rightly pointed out that the electric fields were estimated from the above values in their model.

The purpose of this paper is to design a better model than the model of Hays and Roble (1979) by removing restrictive simplifications which influence the results too much. The main one of these simplifications is taking the integral Pedersen conductivity $\Sigma_{p}$ as a constant for the whole ionosphere. We use the physical model that is only slightly different from Hays and Roble (1979), but, more importantly, more accurate mathematical methods. This allows us to take into account the inhomogeneity of the ionosphere, its Hall conductivity, the singularity of the ionospheric conductivity at the equator, and the nondipolar part of the geomagnetic field. The electric field obtained in our model is about $3 \%$ of that in the model of Hays and Roble (1979).

First, this means that the ionosphere is a much better conductor than was previously considered. Secondly, satellite measurements of the ionospheric electric field that provides closure of the GEC are impossible in practice. Jansky and Pasko (2014) emphasized the importance of an accurate model of the highly conducting ionosphere for the correct description of fast processes like cloud-to-ground lightning discharges, and so such a model is necessary as an element in more complicated models. 
Thus, the objective of the paper is to create a model of electric fields and currents, which constitute the ionospheric part of the GEC. The ionosphere, atmosphere and lithosphere are considered as a continuous conductor, but the many orders of magnitude difference in the conductivities of these three objects makes it possible to split the problem into three separate subproblems and to solve each of them separately.

Section 2 formulates the basics for our model electric conductivity equation, which describes the electric fields and currents in the global conductor under consideration. Section 3 represents the boundary condition for the atmospheric electric field, which replaces the detailed consideration of currents flowing in the lithosphere. Section 4 is devoted to the description of the decomposition method, which allows us to divide the solution of the problem into two problems, in the atmosphere and in the ionosphere.

In Sects. 5-8, we present our model of the atmospheric conductor. In Sect. 5, we simplify the atmospheric problem, approximately considering the currents as being vertical ones. As a consequence, in Sect. 6 we obtain a 1-D model for the electric field and current in each vertical column of the atmosphere. Section 7 describes the altitude dependence of atmospheric conductivity, constructed on the basis of three empirical models. After solving 1-D problems for each atmospheric vertical column and calculating its resistance, the 3-D problem is reduced to a 2-D problem. The input parameter for this is the global distribution of vertical atmospheric conductivity, constructed in Sect. 8. This takes into account topography of the Earth's surface, i.e. its relief, and the presence of oceans and clouds. The resistance of the atmosphere as a whole is calculated. Section 9 specifies the global distribution of thunderstorm generators of vertical electric current, borrowed from the model (Hays and Roble 1979).

The ionospheric part of our model is considered in Sects. 10-14. Section 10 presents our model of the spatial distribution of the components of the conductivity tensor in the ionosphere above $90 \mathrm{~km}$ and the way it interfaces with the atmospheric conductivity below $50 \mathrm{~km}$. Section 11 reduces the 3-D boundary value problem of the electrical conductivity in the ionosphere to the 2-D boundary value problem using a small-parameter expansion. Such a small-parameter is the ratio of the conductivity across the magnetic field to the field-aligned conductivity. On the basis of the charge conservation law, a 2-D equation for the distribution of the electric potential is formulated. It takes into account the presence of the conjugate ionosphere in the opposite hemisphere, thunderstorm currents from the atmosphere, and the fair weather currents. The input parameters for the 2-D equation are the global distribution of the current density from the atmosphere into the ionosphere, constructed in Sect. 9, and the global distributions of the integral Pedersen and Hall conductances, maps of which are constructed in this section.

Section 12 completes the constructed 2-D equation with boundary conditions. Approximate simulation of the main magnetospheric conductors permits us to consider the auroral zones as equipotentials. As a consequence, the ionospheric problem splits into a problem for the main part of the ionosphere, including middle and low latitudes, and two problems for the polar caps. Thus, for the main part of the ionosphere and for the polar caps, three boundary value problems are obtained, each of which has a unique solution. Our numerical method which is used to solve these problems is briefly described.

Section 13 presents and analyzes the ionospheric electric fields and currents obtained as a result of these calculations. Since many of the input parameters of the model either vary with time, or are not well known, Sect. 14 is devoted to an analysis of the effect of these variations and uncertainties on the results obtained. The final Sect. 15, along with a listing of the main results of the presented research, contains an analysis of possible modifications of this model. 


\section{The Electric Conductivity Equation}

Here we regard the atmosphere, ionosphere and magnetosphere as an integrated conductor with the only generator that provides vertical electric current in the atmosphere.

It is adequate to use a steady-state model for a conductor with the conductivity tensor $\hat{\sigma}$ if the typical time of the process is much larger than the charge relaxation time $\tau=\varepsilon_{0} / \sigma$ (Molchanov and Hayakawa 2008). Since atmospheric conductivity increases with height, it has a minimum value near the ground, where $\sigma>10^{-14} \mathrm{~S} / \mathrm{m}$ (Molchanov and Hayakawa 2008). So the charge relaxation time in the Earth's atmosphere is less than a quarter of an hour and such a model can be used for atmospheric electric fields which are not substantially varied during an hour or more. Simulations in the frame of a time-dependent global electric circuit model (Jansky and Pasko 2014) showed that charges produced by lightning relax to zero with the same time scale.

The basic equations for the steady-state electric field $\mathbf{E}$ and current density $\mathbf{j}$ are Faraday's law, the charge conservation law, and Ohm's law,

$$
\begin{gathered}
\operatorname{curl} \mathbf{E}=0, \\
\operatorname{div} \mathbf{j}=Q, \\
\mathbf{j}=\hat{\sigma} \mathbf{E} .
\end{gathered}
$$

Equations $(1,2)$ follow from Maxwell's four equations when all parameters are time independent. Equation (3) is the empirical constitutive equation between $\mathbf{j}$ and $\mathbf{E}$. The given function $Q$ differs from zero if an external electric current exists. Then the total current density is equal to $\mathbf{j}+\mathbf{j}_{\mathrm{ext}}$ and Eq. (2) with $Q=-\operatorname{div} \mathbf{j}_{\mathrm{ext}}$ is the charge conservation law for the total current.

Because of Eq. (1) the electric potential $V$ can be introduced so that

$$
\mathbf{E}=-\operatorname{grad} V .
$$

Then the system of Eqs. (1-3) is reduced to the electric conductivity equation

$$
-\operatorname{div}(\hat{\sigma} \operatorname{grad} V)=Q .
$$

We use spherical geodetic coordinates $r, \theta, \varphi$, spherical geomagnetic coordinates $r, \theta_{m}, \varphi_{m}$, latitude $\lambda=\pi / 2-\theta$, geomagnetic latitude $\lambda_{m}=\pi / 2-\theta_{m}$ and height above mean sea level $h=r-R_{s}(\theta, \varphi)$. The function $R_{s}(\theta, \varphi)$ corresponds to an ellipsoid and will be defined later. Corresponding geodetic and geomagnetic Cartesian coordinates are also used.

\section{Lower Boundary}

The lower boundary of the atmosphere is the Earth's surface. We use the data base (Hastings et al. 1999) that gives height $h_{g}(\theta, \varphi)$ above the mean sea level at the grid in geodetic coordinates $\theta, \varphi$ with step size $0.1^{\circ}$. We use linear interpolation to get height $h_{g}(\theta, \varphi)$ for any $\theta, \varphi$. A diagram of the mean sea level and a mountain is presented in Fig. 1. The mean sea level is the surface $r=R_{s}(\theta, \varphi)$ with function $R_{s}(\theta, \varphi)$ defined in the World Geodetic System WGS 84. This surface is an oblate spheroid (ellipsoid) with radius $a=6378 \mathrm{~km}$ at the equator and the polar radius $b=6357 \mathrm{~km}$ and the mean 
Fig. 1 The ellipsoid $r=R_{s}(\theta, \varphi)$ with half axes $a, b$ - the mean sea level, $h_{g}(\theta, \varphi)$-height of ground, $h$-height of the ionosphere, both above the mean sea level

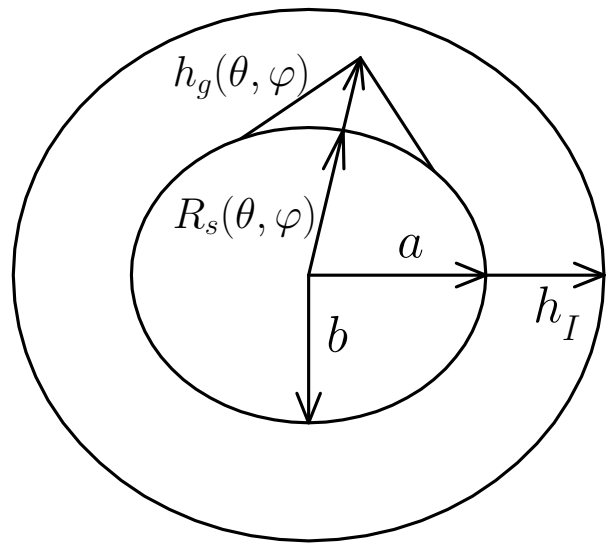

Earth's radius $R_{E} \simeq 6370 \mathrm{~km}$ (World Geodetic System 1984). Our model of the topography is presented in detail in Denisenko and Yakubailik (2015).

The conductivity of the air near ground has a typical value $10^{-14} \mathrm{~S} / \mathrm{m}$. The Earth's nearsurface conductivity ranges from $10^{-7} \mathrm{~S} / \mathrm{m}$ (for poorly conducting rocks) to $10^{-2} \mathrm{~S} / \mathrm{m}$ (for clay or wet limestone), with a mean value of $3.2 \mathrm{~S} / \mathrm{m}$ for the ocean (Rycroft et al. 2008). So the Earth's ground is usually regarded as an ideal conductor, that means a constant value of the electric potential at the Earth's surface

$$
\left.V\right|_{h=h_{g}(\theta, \varphi)}=-V_{0},
$$

where $V_{0}$ is a constant whose value will be defined later.

For fast processes like cloud-to-ground lightning discharges, it is necessary to take the finite conductivity of the ground into account as is done in the model of Jansky and Pasko (2014), but here we study processes with much larger time scales.

\section{Separation of lonospheric and Atmospheric Conductors}

After separating the ground with the help of the boundary condition (6), the remaining conductor consists of the atmosphere, ionosphere and magnetosphere. There are no clear boundaries separating the ionosphere, but we define its conditional boundaries at the altitudes of $h_{I}=90 \mathrm{~km}$ and $h_{M}=500 \mathrm{~km}$ to divide the regions with substantially different conductivities. Ionospheric currents flow across the magnetic field mainly at heights of 80-500 km (Hargreaves 1979). The reason and the possibility to shift the lower boundary from 80 to $90 \mathrm{~km}$ we discuss in Sect. 10.

In the separated ionosphere, the conductivity in the direction of the magnetic field $\mathbf{B}$ exceeds by several orders of magnitude the conductivity in perpendicular directions, which makes it possible to use the 2-D model described in Sect. 11. To simulate the magnetosphere, defined as a region above $500 \mathrm{~km}$, we regard the conductivity in directions perpendicular to $\mathbf{B}$ as zero in Sect. 12.

In this paragraph, we consider the atmosphere as a region below $90 \mathrm{~km}$. However, in Sects. 5 and 6 we additionally separate the layer 50-90 km, where the conductivity differs significantly from being isotropic, in contrast to the underlying lower part of the atmosphere, but there is no dominance of conductivity along $\mathbf{B}$, which occurs 
above $90 \mathrm{~km}$. Because of this, the calculation of the electric fields and currents in the 50-90 km layer would be much more complicated than in the other regions, if it was required. Solving such a complex 3-D problem can be avoided, since this layer has little effect on both the conductivity of the atmosphere in the direction of the groundionosphere (Sects. 5 and 6), and the conductivity of the ionosphere in the horizontal directions (Sect. 11).

To visualize the ionospheric electric fields, we use a surface at an altitude of $h=120 \mathrm{~km}$. This choice is explained in Sect. 11. Since ionospheric conductivity is many orders of magnitude larger than the atmospheric conductivity, the ionosphere can be approximately simulated as an ideal conductor, that means $V(h, \theta, \varphi)=0$ at large heights $h>h_{I}$. Such a conventional height of the ionosphere $h_{I}$ ought to be taken large enough to make only a small influence on the atmospheric electric fields and currents. Test calculations in Ampferer et al. (2010) show that $h_{I}>90 \mathrm{~km}$ is enough. Then the boundary condition takes the shape

$$
\left.V\right|_{h=h_{I}}=0 .
$$

It completes the Dirichlet boundary value problem $(5,6,7)$ for the atmosphere that is simulated as a conductor between two ideal conductors. Such a problem has a unique solution when the constant $V_{0}$ is given. Then current densities through the boundaries can be calculated. Because of the charge conservation law just these currents appear in the ionosphere and under the ground correspondingly. They are to be used in the boundary conditions for two more conductivity problems in the ionosphere and underground. We are not interested in underground fields here and the ionospheric model is described in Sect. 11.

The solutions for these problems give some distributions of potential at the boundaries which differ from constants $V_{0}$ and zero. They can be used as the new right-hand sides in the boundary conditions $(6,7)$ in the atmospheric problem for which a new solution will be more precise. These iterations can be repeated if necessary. Such a method is referred to as domain decomposition. It has fast convergence if conductivities in the separated domains differ greatly. As we will see later, iterations are not necessary for our problem because potential differences in the ionosphere $\delta V(h, \theta, \varphi)$ are three or four orders of magnitude less than $V_{0}$ and so have a negligible influence on the currents from the atmosphere to the ionosphere.

Simulation of the Earth's ionosphere as an ideal conductor is conventional (Rycroft and Odzimek 2010), but it is stressed in the same paper that this is valid only for atmospheric problems. There is the opposite relation for magnetospheric generators. The ionosphere is a bad conductor in comparison with the magnetosphere and so electric fields in the highlatitude ionosphere can be found by mapping along magnetic field lines from distant parts of the magnetosphere.

The variations of the ionospheric potential at high latitudes are mentioned in Tinsley and Zhou (2006) and in many other papers. The penetration of the ionospheric electric fields into the atmosphere is analyzed in Denisenko et al. (2009) and Roble and Hays (1979). It can be analyzed separately in view of the linearity of the problem and these fields constitute a part of the GEC that is not generated by thunderstorms. Moreover, the influence of magnetospheric generators cannot be regarded as a part of the GEC if this circuit is closed in the atmosphere, ionosphere and ground as is shown in Fig. 1 in Tinsley and Zhou (2006). Strictly speaking the $100 \mathrm{kV}$ potential difference in the ionosphere shown in that diagram could not exist without currents from the magnetosphere which are not shown. This definition of the GEC is a conventional one as is shown in the review of Mareev (2010). So we do not include these fields and currents into the actual model. 
$\mathbf{a}$

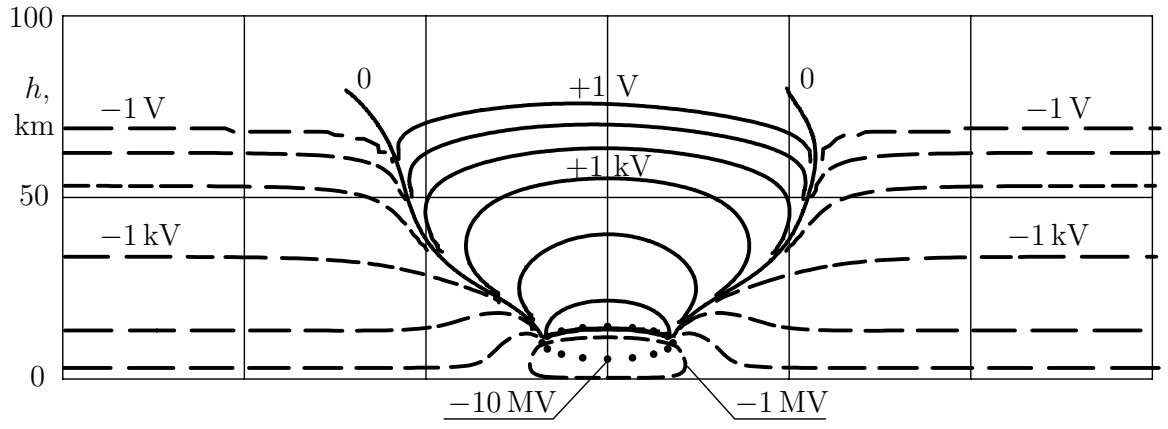

b

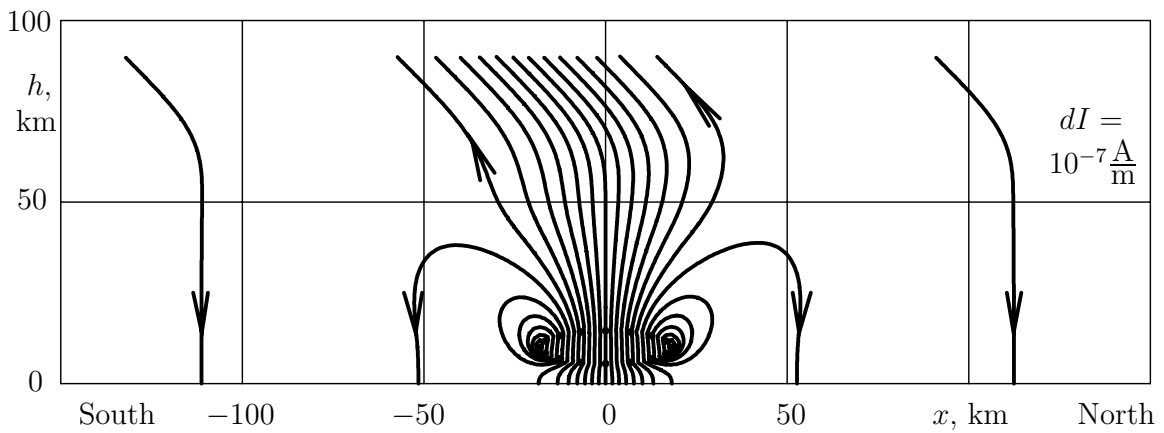

Fig. 2 The equipotentials (a) and the current lines for the total current (b) in the neighborhood of a thunderstorm cloud. Dots show elliptical cross sections of the cloud. Figure is reproduced from Denisenko (2014b)

\section{The Currents in the Atmosphere}

The electric fields and currents in the neighborhood of a thunderstorm cloud were calculated in the paper (Denisenko 2014b) within the same model of the electrical conductivity, which we use in this paper, with $\sigma(h)$ distribution (Rycroft and Odzimek 2010). A cloud elongated by the magnetic longitude was studied. So a two-dimensional model was used. All parameters do not depend on the magnetic longitude, including the magnetic inclination $\alpha$.

The cloud is located at altitudes $5-15 \mathrm{~km}$ and it has a width $40 \mathrm{~km}$. Its cross section is the ellipse shown by a thin line in Fig. 2a. Inside the cloud according to Rycroft and Odzimek (2010) the conductivity was decreased 10 times compared to the conductivity at the same height outside the cloud. This change occurs in a narrow boundary layer between the two ellipses shown by the thin lines in Fig. 2a. In the interior part of the cloud, a uniform vertical current with density $j_{\text {ext }}^{\text {cloud }}=110 \mathrm{pA} / \mathrm{m}^{2}$ was defined. It decreased to zero in the boundary region. This current density was selected such that a potential difference of about $10 \mathrm{MV}$ arose between the upper and lower boundaries of the cloud. The potential difference between the ionosphere and the ground was taken as $V_{0}=345 \mathrm{kV}$ in that model, $V=0$ in the ionosphere, $-V_{0}$ at ground. It was chosen so that the fair weather electric field near the ground was $E_{0}=130 \mathrm{~V} / \mathrm{m}$. The corresponding fair weather current density was $j_{0}=1.7 \mathrm{pA} / \mathrm{m}^{2}$. 
A numerical solution for this electroconductivity problem was obtained. The resulting electric field for $\alpha=45^{\circ}$ is presented in Fig. 2a in the meridional plane. The equipotentials are plotted on a logarithmic scale: the values at adjacent lines differ by a factor of 10 . Only the zero line is shown for $|V|<1 \mathrm{~V}$. Above $h=80 \mathrm{~km}$ the potential differences are $<0.1 \mathrm{~V}$.

Figure $2 \mathrm{~b}$ shows the current lines for the total current $\mathbf{j}_{\text {tot }}=\mathbf{j}_{\text {ext }}^{\text {cloud }}+\hat{\sigma} \mathbf{E}$. Below $h=50 \mathrm{~km}$ the inclination of the magnetic field has no effect. The distributions of electric fields and currents are the same as in the case of the vertical magnetic field which is also discussed in Denisenko (2014b). Above $h=50 \mathrm{~km}$ the currents shift horizontally by only 20-30 km from being vertically above the cloud, and this property is maintained for wider clouds. Above $h=50 \mathrm{~km}$ the current lines, remaining almost parallel to each other, turn and above $h=70 \mathrm{~km}$ they almost take the direction of the magnetic field $\mathbf{B}$. The directions of the fair weather currents appear to be rather simple: they are vertical below $\simeq 60 \mathrm{~km}$ and go along $\mathbf{B}$ above $\simeq 70 \mathrm{~km}$.

It is demonstrated in Denisenko (2014b) that for the clouds with width $>100 \mathrm{~km} \mathbf{j}_{\text {tot }} \simeq 0.6 \mathbf{j}_{\text {ext }}^{\text {cloud }} \simeq 70 \mathrm{pA} / \mathrm{m}^{2}$ and the current density is independent of the height. These properties correspond to a 1-D model described in the next section.

From the described model (Denisenko 2014b) for us only the following property of the large-scale currents is important. Thunderstorm generated currents appear in the ionosphere with a horizontal shift of only a few tens of $\mathrm{km}$ when they rise from the lower atmosphere. These currents, as well as fair weather currents, also are shifted horizontally in the direction of the magnetic field by $\delta x \simeq 20 \mathrm{~km} / \tan (\alpha)$. For example, the Himalayas with such a projection into the ionosphere must be shifted to the South by $\delta x \simeq 30 \mathrm{~km}$.

Near the magnetic equator, the shift is not described by this formula. Thunderstorm clouds at the geomagnetic equator are similarly considered in Denisenko (2014a). The above-described shift to the north or to the south in such a case disappears because of the symmetry, and expansion during the flow up through the atmosphere reaches $\simeq 100 \mathrm{~km}$. The shift to the east for a distance about $100 \mathrm{~km}$ appears because of the Hall conductivity.

In this model, we have neglected all these displacements and extensions because we are interested only in horizontal scales $>>100 \mathrm{~km}$. We also neglect the electric fields above $h=50 \mathrm{~km}$, which provide the passage of the currents from this height to the ionosphere, because the potential difference is less than $0.1 \%$ of the potential difference below this height.

To this accuracy, we consider the currents in the atmosphere as vertical ones. Such an approximation is analyzed in Slyunyaev et al. (2015). Then atmospheric electric fields are vertical, too, because the conductivity is scalar below $h=50 \mathrm{~km}$. It is shown in Ampferer et al. (2010) that for the events with horizontal scale $<100 \mathrm{~km}$ the atmospheric currents are no longer purely vertical. So our model would not be accurate if applied for simulation above mountains with steep slopes and peaks.

We substitute a detailed consideration of the external currents inside thunderclouds with vertical charge transfer from ground to the ionosphere as is shown in Fig. 3. Let us separate a vertical column of the atmosphere with cross section $1 \mathrm{~m}^{2}$. The left panel presents three parts of such a conductor with resistances $\rho_{a}$ between ground and lower boundary of a cloud, $\rho_{c}$ inside a cloud, and $\rho_{i}$ between a cloud and the ionosphere. A generator of external current $j_{\text {ext }}^{\text {cloud }}$ works inside the cloud. Just the density of the external current is the original physical parameter while the voltage and the charge density inside a cloud are the results (Feynman et al. 1964). So we use it as the given value in the model of separate clouds (Denisenko 2014b) for which results are shown in Fig. 2 and in the actual global model.

The ionosphere has zero potential and the ground potential equals $-V_{0}$. It is simple to calculate the parameters $\rho=\rho_{a}+\rho_{c}+\rho_{i}$ and $j_{\mathrm{ext}}=j_{\mathrm{ext}}^{\text {cloud }} \rho_{c} / \rho$ of the equivalent electric circuit 
Fig. 3 Circuit diagram of the atmospheric vertical column with $1 \mathrm{~m}^{2}$ cross section between the ionosphere $(V=0)$ and ground $\left(V=-V_{0}\right)$ including a thunderstorm cloud. A thunderstorm generator of the external current $j_{\text {ext }}^{\text {cloud }}$ inside a cloud and three resistors (left panel) are equivalent to some generator and resistor-right panel

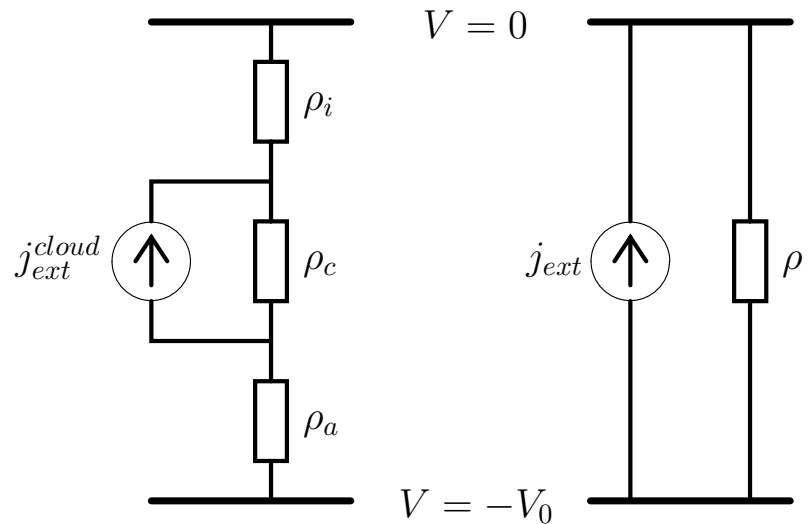

shown in the right panel. The current through the resistor $\rho$ is considered together with other conduction currents in the frame of the boundary value problem $(5,6,7)$. A similar approach is used by Slyunyaev et al. (2015).

Of course, there are large currents due to transient sprites but their effect on the global atmospheric electric circuit is small (Fullekrug and Rycroft 2006). Here we suppose that the parameter $\rho$ is defined only by the steady-state conductivity without its short duration increases due to sprites and lightning discharges when relations between $j_{\mathrm{ext}}^{\text {cloud }}$ and $j_{\mathrm{ext}}$ may be much more complicated. In Sect. 10, we construct the global distribution of the effective external current density $j_{\text {ext }}$ in accordance with the empirical model of Hays and Roble (1979); only this is the input parameter for our model. Its integral over all the Earth's surface we designate as

$$
I_{e x t}=\int j_{\mathrm{ext}}(\theta, \varphi) r^{2} \sin \theta \mathrm{d} \theta \mathrm{d} \varphi
$$

where $r=R_{s}(\theta, \varphi)+h_{g}(\theta, \varphi)$.

\section{1-D Model of the Atmospheric Conductor}

In accordance with the analysis made in the previous section for atmospheric conduction currents we use the flat 1-D model instead of 3-D equation (5) with boundary conditions $(7,6)$

$$
-\frac{\mathrm{d}}{\mathrm{d} h}\left(\sigma(h) \frac{\mathrm{d}}{\mathrm{d} h} V(h)\right)=0,\left.\quad V\right|_{h=h_{0}}=0,\left.\quad V\right|_{h=h_{g}(\theta, \varphi)}=-V_{0} .
$$

It also was shown in that section that the conductivity above $h=50 \mathrm{~km}$ is so large that the potential difference above $h=50 \mathrm{~km}$ is less than $0.1 \%$ of the potential difference below this height. So we shift the boundary condition (7) from the height $h_{I} \geq 90 \mathrm{~km}$ to $h_{0}=50 \mathrm{~km}$ in (9). Rather than the tensor form, this permits us to use the scalar conductivity $\sigma(h)$ for calculations of the electric fields and currents in the atmospheric part of the global conductor.

We use the equation for a flat model (9) instead of its spherical version

$$
-\frac{1}{r^{2}} \frac{\mathrm{d}}{\mathrm{d} r}\left(r^{2} \sigma\left(r-R_{s}(\theta, \varphi)\right) \frac{\mathrm{d}}{\mathrm{d} r} V(r)\right)=0 .
$$


This equation is equivalent to the equation in (9) if its coefficient $\sigma(h)$ is multiplied by $\left(r /\left(R_{s}(\theta, \varphi)+h_{0} / 2\right)\right)^{2} \simeq 1+\left(2 h-h_{0}\right) / R_{s}(\theta, \varphi)$, and this multiplier negligibly differs from 1 by an amount $\pm h_{0} / R_{s}(\theta, \varphi) \simeq \pm h_{0} / R_{E} \simeq 0.01$.

The solution $V(h)$ of the problem (9) must have indices $\theta, \varphi$ since the input values depend on coordinates. For example, in a cloudy area the conductivity of the atmosphere is reduced as was described in the previous section. We omit the indices $\theta, \varphi$ for brevity. The solution to this problem gives the strength of the vertical electric field $E(h)=-\mathrm{d} V / \mathrm{d} h$ and current density $j=-\sigma(h) \mathrm{d} V / \mathrm{d} h$.

By virtue of Eq. (9), the current density $j$ does not vary with height, and hence $j$ is a function only of the coordinates $\theta, \varphi$. The last property allows us to reduce the solution of the problem (9) to the integration over height:

$$
-V_{0}=j(\theta, \varphi) \int_{h_{g}(\theta, \varphi)}^{h_{0}} \frac{\mathrm{d} h}{\sigma(h)} .
$$

The last integral is the resistance of the atmospheric vertical column with $1 \mathrm{~m}^{2}$ cross section between ground and ionosphere

$$
\rho(\theta, \varphi)=\int_{h_{g}(\theta, \varphi)}^{h_{0}} \frac{\mathrm{d} h}{\sigma(h)} .
$$

It is not difficult to calculate the integral of the given function numerically. Then we get the value $j(\theta, \varphi)$ from Eq. (10). The inverse value

$$
\Sigma(\theta, \varphi)=1 / \rho(\theta, \varphi)
$$

is the conductance of the same atmospheric vertical column.

\section{Height Distribution of Conductivity in the Atmosphere}

Thin lines in Fig. 4 show the height distributions of the conductivity $\sigma(h)$, proposed for fair weather above ground by the empirical models (Rycroft and Odzimek 2010; Handbook of Geophysics 1960; Molchanov and Hayakawa 2008). The real values of $\sigma$ can be several times different from the model ones (Handbook of Geophysics 1960). The model (Handbook of Geophysics 1960) is based on direct measurements, but the statistical variance of the values does not contradict the other mentioned models. Since we do not have strong arguments in favor of one of these models, in this paper we use the averaged distribution shown by the bold line in the same figure. It is constructed in such a way that it does not contradict the three models and satisfies two conditions: $\sigma(0)=1.54 \times 10^{-14} \mathrm{~S} / \mathrm{m}$, $\rho(0)=1.25 \times 10^{17} \Omega \mathrm{m}^{2}$. The first condition ensures, under fair weather, a vertical current density $j_{0}=2 \mathrm{pA} / \mathrm{m}^{2}$ when the vertical electric field strength equals $E_{0}=130 \mathrm{~V} / \mathrm{m}$. The second condition by virtue of (10) provides a potential difference between ground and the ionosphere $V_{0}=250 \mathrm{kV}$ for the same $E_{0}$. The given values of $E_{0}, j_{0}, V_{0}$ are considered to be typical for the GEC (Rycroft et al. 2008). 
Fig. 4 Models of the height distributions of the conductivity for fair weather above ground. Thin lines: 1-Rycroft and Odzimek (2010), 2-Handbook of Geophysics (1960), 3-Molchanov and Hayakawa (2008). Bold line-our model that is designed as some average

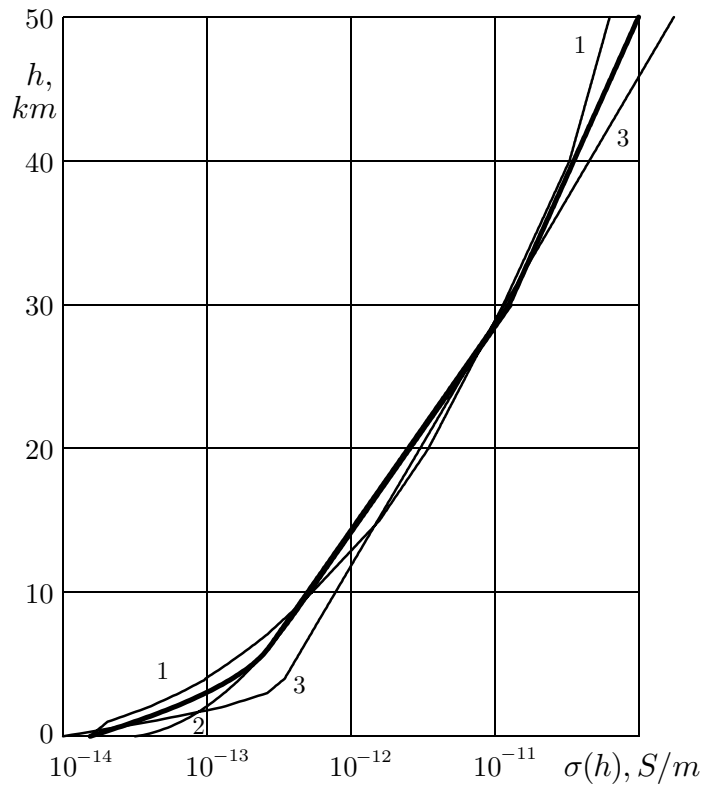

The constructed function $\log _{10}(\sigma(h))$ is given by the following formulae:

$$
\begin{aligned}
& s_{30}+(h-30)\left(s_{50}-s_{30}\right) / 20, \quad 30 \leq h \leq 50, \\
& -13+h\left(s_{30}+13\right) / 30, \quad 6.5 \leq h \leq 30, \\
& -13+h\left(s_{30}+13\right) / 30-(h / 6.5-1)^{2}\left(s_{0}-13\right), \quad h \leq 6.5,
\end{aligned}
$$

where the values at $h=50,30,0 \mathrm{~km}$ are $s_{50}=-10, s_{30}=-10.9, s_{0}=-13.812$ for ground or $s_{0}=-13.51$ for sea.

A cloud changes the conductivity $\sigma(h)$ obtained by these formulae only at $5<h<15 \mathrm{~km}$; the cloud conductivity is the clear air conductivity multiplied by at $6 \leq h \leq 14 \mathrm{~km}$ with linear interpolation of this multiplier near the cloud's boundaries at $5<h<6 \mathrm{~km}$ and at $14<h<15 \mathrm{~km}$.

Of course, it is a very simplified model. Measurements (Handbook of Geophysics 1960) show tenfold variations of conductivity near the ground. We also suppose that mountains do not vary the properties of the surrounding air, while really they increase the amount of aerosols and so decrease the conductivity of the air. The aerosol effect on the conductivity, may, however, be partially compensated by their contribution of additional radioactive ionization from their surface geology.

\section{Global Distribution of Conductivity in the Atmosphere}

In our model, $\rho(\theta, \varphi)$ is determined by three factors. The first of them is the height of the Earth's surface $h_{g}(\theta, \varphi)$ that is the lower limit of integration in (11).

The resistance $\rho$ decreases with increasing altitude of ground surface. A plot of $\rho$ as a function of the height of ground surface $h=h_{g}(\theta, \varphi)$ is shown in Fig. 5. It corresponds to 
Fig. 5 Resistance of the atmospheric vertical column with $1 \mathrm{~m}^{2}$ cross section between the ionosphere and ground that is at a height $h$ above the sea level. The curves "cloud" represent the cases when the conductivity at heights $h=5-15 \mathrm{~km}$ is decreased. The curves "ground" show the resistances above ground with and without clouds. The curves "sea" show the resistances above sea with and without clouds

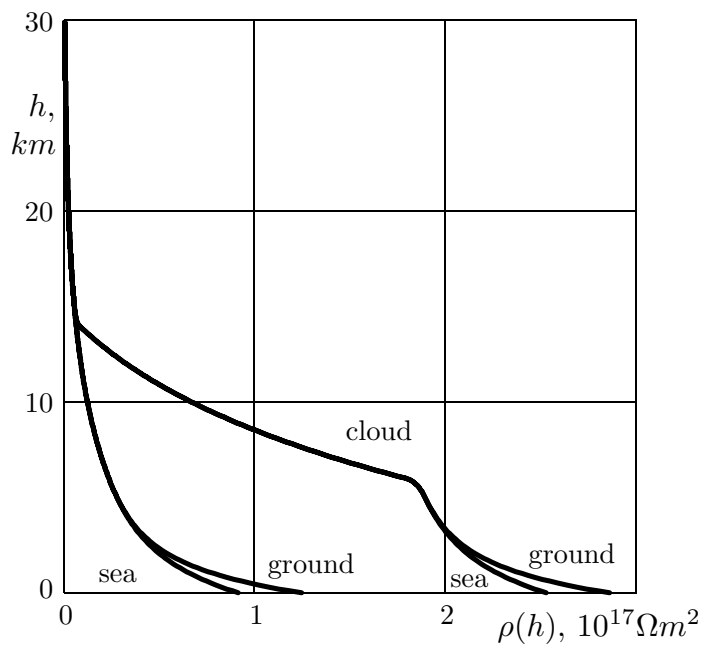

Fig. 6 in Rycroft et al. (2008). For example, both figures show that the resistance of the layer $0-1.7 \mathrm{~km}$ is equal to that of the layer $1.7-10 \mathrm{~km}$. We can see that the atmosphere above $30 \mathrm{~km}$ does not contribute significantly.

The second reason for $\rho(\theta, \varphi)$ variations is the increased conductivity in the lower atmosphere above the sea. According to Molchanov and Hayakawa (2008), the conductivity above the sea surface is twice as high as above ground, and this difference disappears at an altitude of $2-4 \mathrm{~km}$. Since the surface layer, in which the conductivity rapidly increases with altitude, in our model, as well as in other models (Rycroft and Odzimek 2010; Kudintseva et al. 2016; Handbook of Geophysics 1960), is substantially smoother in comparison with model of Molchanov and Hayakawa (2008), we make the difference between the conductivities above the ground and sea equal to zero above $6.5 \mathrm{~km}$, while it is negligible above $4 \mathrm{~km}$.

The third modification is due to the decrease in conductivity inside clouds. In accordance with the model of Rycroft and Odzimek (2010), due to cloudiness, the conductivity decreases by a factor of 5-10 at altitudes of 5-15 km. We reduce the conductivity by a factor of 10 at altitudes of 6-14 km with a linear interpolation to values outside the cloud in the 5-6 km and 14-15 km layers. So the total resistance from the ground at sea level to the ionosphere increases by a factor of two as the curves "cloud" in Fig. 5 show. The curves "sea" and "ground" show the resistances above the sea and ground correspondingly. They are plotted for the atmosphere with and without clouds. The resistances $\rho_{a}$, $\rho_{c}$, and $\rho_{i}$, which are, respectively, the resistances between ground and lower boundary of a cloud, inside a cloud, and between a cloud and the ionosphere, are studied separately in the model (Slyunyaev et al. 2015) and so the ratios of their model values are calculated. They are $\rho_{c} / \rho_{i}=2.8, \rho_{a} / \rho_{i}=3.6$ if a cloud is between $h=4 \mathrm{~km}$ and $h=12 \mathrm{~km}$. Figure 5 shows these ratios equal to 2.7 and 6.3 for fair weather conditions above sea in our model and $\rho_{a} / \rho_{i}=10$ above ground. It means that above $h=4 \mathrm{~km}$ our models do not differ, and below $h=4 \mathrm{~km}$ the conductivity in the model of Slyunyaev et al. (2015) does not decrease near ground so much as for our model shown in Fig. 4. For example, $\sigma(0)=1.54 \times 10^{-14} \mathrm{~S} / \mathrm{m}$ and $3 \times 10^{-14} \mathrm{~S} / \mathrm{m}$ correspondingly above ground and sea in our model and $\simeq 5.5 \times 10^{-14} \mathrm{~S} / \mathrm{m}$ in Slyunyaev et al. (2015). 
We suppose that the density of clouds $C_{\mathrm{cl}}$ is proportional to the external current density $j_{\text {ext }}\left(\theta_{m}, \varphi_{m}\right)$ using the model which is described in the next section. Outside thunderstorm areas $C_{\mathrm{cl}}=0$, and it would reach $C_{\mathrm{cl}}=1$ if $j_{\mathrm{ext}}=60 \mathrm{pA} / \mathrm{m}^{2}$. Such a value is rather arbitrary and we will discuss its influence on the results later. Since $j_{\mathrm{ext}}$ defined in the next section has a maximum value of about $60 \mathrm{pA} / \mathrm{m}^{2}$ in central Africa, our definition of $C_{\mathrm{cl}}$ means permanent clouds inside that area. We do not include other clouds in the model.

The total current through the atmosphere can be obtained by integrating over all the Earth's surface:

$$
I=\int j(\theta, \varphi) r^{2} \sin \theta \mathrm{d} \theta \mathrm{d} \varphi
$$

where $r=R_{s}(\theta, \varphi)+h_{g}(\theta, \varphi)$.

Due to the linearity of the problem (9) with respect to $V_{0}$, the total current $I$ is also proportional to $V_{0}$. The resistance $R$ of the atmosphere as a whole including the regions of thunderstorms with decreased conductivity is defined by the relation

$$
-V_{0}=R I \text {. }
$$

In view of $(10,13)$ it can be calculated as

$$
\frac{1}{R}=\int \Sigma(\theta, \varphi) r^{2} \sin \theta \mathrm{d} \theta \mathrm{d} \varphi .
$$

The signs are chosen so that for positive values of $V_{0}$ the Earth has a negative potential with respect to the ionosphere, and the electric field $E$ and the current $j$ are directed downwards. Therefore, the total current $I$ is also negative and the resistance $R$ is positive. As the result of integration of (15), we have $R=180 \Omega$.

The typical value $R=190 \Omega$ with an increase up to $R=300 \Omega$ in some models is presented in Tinsley and Zhou (2006). Many factors like volcanos and radon production from the ground are analyzed in those models. Our model is not so sophisticated because we are interested mainly in the ionospheric part of the GEC. Anyway we can vary the model value of $R$ between certain limits by the variation of the density of clouds. If clouds are everywhere the resistance $R_{3}=496 \Omega$. If clouds are absolutely absent $R_{2}=175 \Omega$. So the presence of clouds could vary the total resistance of the atmosphere by about 2.8 times. Now we take into account only a small portion of the clouds, namely thunderclouds.

If the Earth is a sphere without seas $R_{0}=245 \Omega$ that corresponds to the fair weather value $\rho_{0}=1.25 \times 10^{17} \Omega \mathrm{m}^{2}$ for our model of conductivity. If the surface of the Earth were at the sea level, $h_{g}(\theta, \varphi)=0$ everywhere and no cloud exists, these formulae would give $R_{1}=195 \Omega$ instead of $R_{2}=175 \Omega$. Thus, the Earth's relief reduces the total resistivity of the atmosphere by about $11 \%$. The model of Jansky et al. (2017) gives $R=235 \Omega$ with a decrease by $10 \Omega$ because of topography. Such a decrease in our model is twice as large because we use a conductivity profile with a boundary layer near the ground as is shown in Fig. 4. The local resistance of a vertical column of the fair weather atmosphere over the high mountains is reduced much more, about 5 times over the mountains with $6 \mathrm{~km}$ height as is shown in Figs. 5 and 6. The model (Baumgaertner et al. 2014) gives up to twice larger column resistance because of clouds. Possible results within the framework of our model we discuss in Sect. 14.

A total atmospheric resistance of $227 \Omega$ was obtained from the modeling work of Makino and Ogawa (1985), increasing to $258 \Omega$ with a $20 \%$ decrease in cosmic ray ionization or $242 \Omega$ for a $20 \%$ increase in global aerosol burden. For this evaluation Makino and Ogawa 


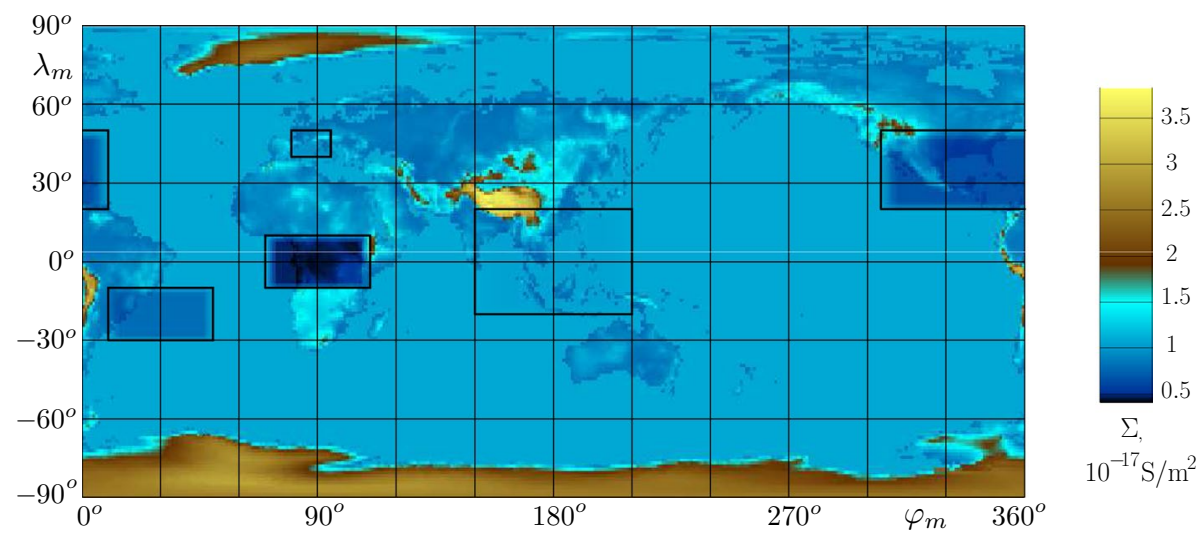

Fig. 6 The global distribution of the vertical conductance of the atmosphere $\Sigma\left(\lambda_{m}, \varphi_{m}\right)$ in units of $10^{-17} \mathrm{~S} / \mathrm{m}^{2}$. The rectangles show the regions with electric current to the ionosphere from thunderstorm clouds (Hays and Roble 1979)

(1985) assumed an equipotential ionosphere, with the spatial variation in columnar resistance found by combining the cosmic ray ionization profile with aerosol concentrations chosen to represent conditions above the continents and oceans. [The detailed representation chosen of the aerosol profile, clouds and cosmic ray ionization determines the exact value of the total atmospheric resistance obtained from such modeling (Baumgaertner et al. 2014) .] Above the Tibetan plateau, Makino and Ogawa (1985) found that the columnar resistance was $2.7 \times 10^{16} \Omega \mathrm{m}^{2}$, compared with $1.3 \times 10^{17} \Omega \mathrm{m}^{2}$ over the Indian ocean, which is again a factor of about 5 between the highest mountain altitudes and sea level.

The distribution of the vertical columnar conductance of the atmosphere in geomagnetic coordinates $\Sigma\left(\lambda_{m}, \varphi_{m}\right)$ is shown in Fig. 6. As can be seen in Fig. $4 \Sigma=1.1 \times 10^{-17} \mathrm{~S} / \mathrm{m}^{2}$ above sea without clouds and $0.8 \times 10^{-17} \mathrm{~S} / \mathrm{m}^{2}$ above ground at sea level. So the shore lines correspond to $\Sigma=10^{-17} \mathrm{~S} / \mathrm{m}^{2}$ in Fig. 6. The average value of $\Sigma$ is about $1.1 \times 10^{-17} \mathrm{~S} / \mathrm{m}^{2}$ and $\Sigma$ is increased above mountains. These features are also described in Rycroft et al. (2000) and Tinsley and Zhou (2006). The main features of Fig. 6 look similar to those in Fig. 14 in Tinsley and Zhou (2006).

\section{Global Thunderstorm Generator}

We use the model (Hays and Roble 1979) of the global distribution of thunderstorm activity. They separate five main thunderstorm regions as typical ones for a northern hemisphere summer at 19:00 UT. These regions are defined as quadrangles in geomagnetic coordinates $\lambda_{m}, \varphi_{m}$. They are plotted in Fig. 6. It is supposed in Hays and Roble (1979) that all thunderstorm generators produce $2 \mathrm{kA}$ total vertical current above clouds that is distributed in these five regions proportionally to the number of thunderstorms in them, namely $600 \mathrm{~A}$ in Africa, $100 \mathrm{~A}$ is Southern Asia, $1000 \mathrm{~A}$ in Central America, 280 A in Southern America, $20 \mathrm{~A}$ in Europe.

By virtue of the charge conservation law, the total external current (with the opposite sign) is equal to the total current of atmospheric conductivity $I(13)$, which is provided by the potential difference between ground and ionosphere (14). The total resistance of the 
atmosphere for the conductivity model used is found above as $R=180 \Omega$ (15). The current $I=2 \mathrm{kA}$ corresponds to $360 \mathrm{kV}$.

We believe that the parameter $V_{0}$ is known more accurately than $I_{\text {ext }}$. Therefore, we use as a given value of $V_{0}=250 \mathrm{kV}$ (Rycroft et al. 2008) and calculate the total conductivity current from Ohm's law (14) with the value $R=179 \Omega$ that is found above

$$
I=-V_{0} / R=-1.4 \mathrm{kA},
$$

where the minus corresponds to the downwards direction. To fulfill the charge conservation law when $I_{\mathrm{ext}}=-I=V_{0} / R=1.4 \mathrm{kA}$ we reduce the density of external currents (Hays and Roble 1979) by 1.43 times. Then the currents in the five rectangles become equal to $420 \mathrm{~A}, 70 \mathrm{~A}, 700 \mathrm{~A}, 196 \mathrm{~A}$, and $14 \mathrm{~A}$, respectively.

So the total current of the GEC $I_{\text {ext }}=-I=1.4 \mathrm{kA}$ in our model. It is possible to increase the current up to $2 \mathrm{kA}$ in the model if we increase $V_{0} 1.43$ times, when the key parameters $E_{0}, j_{0}$ are also increased. As an alternative we can use another model of atmospheric conductivity. For example, the model (Molchanov and Hayakawa 2008) presented with curve 3 in Fig. 4 gives the total resistance of the atmosphere $129 \Omega$ instead of our $R=179 \Omega$ and so there would be $I=1.94 \mathrm{kA}$ for the same $V_{0}=250 \mathrm{kV}$. Since $\sigma(0)=10^{-14} \mathrm{~S} / \mathrm{m}$ and $\rho(0)=10^{-17} \mathrm{~S} / \mathrm{m}^{2}$ in that model, the fair weather electric field and current would be $E_{0}=250 \mathrm{~V} / \mathrm{m}, j_{0}=2.5 \mathrm{pA} / \mathrm{m}^{2}$.

It is more simple to decrease the total current. If the density of clouds $C_{\mathrm{cl}}=0.465$ all over the globe $\left(C_{\mathrm{cl}}=1\right.$ would mean total cloud cover) the total resistance of the atmosphere equals $250 \Omega$ instead of our $R=179 \Omega$ and so there would be $I=1 \mathrm{kA}$ for the same $V_{0}=250 \mathrm{kV}$ with the conventional fair weather surface electric field $E_{0}=130 \mathrm{~V} / \mathrm{m}$.

We next somewhat smooth out the distribution of $j_{\mathrm{ext}}\left(\lambda_{m}, \varphi_{m}\right)$ in comparison with Hays and Roble (1979). Instead of having a jump from zero to a value that is constant inside each of five rectangles, we construct linear functions in each strip near the boundary whose width is equal to one tenth of the side of the rectangle. Such smoothing does not matter from the physical point of view, since the model of $j_{\text {ext }}\left(\lambda_{m}, \varphi_{m}\right)$ is too rough, but it improves the properties of the problem from the computational point of view.

\section{Conductivity in the Earth's Atmosphere and lonosphere}

We use parallel and normal to the direction of magnetic induction $\mathbf{B}$ components of vectors which are marked with symbols $\|$ and $\perp$. Then Ohm's law (3) in a gyrotropic medium takes the form

$$
j_{\|}=\sigma_{\|} E_{\|}, \quad \mathbf{j}_{\perp}=\sigma_{P} \mathbf{E}_{\perp}-\sigma_{H}\left[\mathbf{E}_{\perp} \times \mathbf{B}\right] / B,
$$

with Hall $\left(\sigma_{H}\right)$ Pedersen $\left(\sigma_{P}\right)$ and field-aligned $\left(\sigma_{\|}\right)$conductivities (Kelley 2009).

We have created the model (Denisenko et al. 2008) to calculate the components $\sigma_{P}, \sigma_{H}$, $\sigma$ of the conductivity tensor $\hat{\sigma}$ above $h=90 \mathrm{~km}$, that is based on the empirical models IRI, MSISE, IGRF. In that model, the ionospheric conductivity is calculated up to an altitude of $2000 \mathrm{~km}$. For our calculations, we use the profile up to the top of the ionospheric F-layer at $h_{M}=500 \mathrm{~km}$. If we include the layer above this height the parameters of interest which are integral Pedersen and Hall conductivities would increase by only $1 \%$.

The model IRI gives values of the electron concentration for $h>80 \mathrm{~km}$ for the night and for $h>60 \mathrm{~km}$ for the day. Some irregular structures appear at $80 \mathrm{~km}<h<90 \mathrm{~km}$ and IRI 
is not precise enough to describe them. For our purpose, the interval $80 \mathrm{~km}<h<90 \mathrm{~km}$ is not important for two reasons. Its presence negligibly varies the conductance of the atmospheric column because the conductivity there is many orders of magnitude larger than below $30 \mathrm{~km}$. In Sect. 11, we show that the layer $h<90 \mathrm{~km}$ only slightly varies the integral conductance of the ionosphere. So it is enough to use below $90 \mathrm{~km}$ the following simplified model of conductivity.

Below $50 \mathrm{~km}$, we use the model constructed above in Sect. 6. It is close to the empirical model of Rycroft and Odzimek (2010). The electric conductivity is isotropic there. It does not depend on the magnetic field and so we can identify it as the field-aligned $\sigma_{\|}$conductivity. At the heights $h=50-90 \mathrm{~km}$ the transformation from an atmospheric type of variation to an ionospheric one occurs (Rycroft and Odzimek 2010; Schlegel and Fullekrug 2002). We approximate a height dependence in the upper atmosphere as a smooth continuation from the ionosphere above $h=90 \mathrm{~km}$ to the values below $50 \mathrm{~km}$ which are typical for the atmosphere. Namely, in the layer $50 \mathrm{~km}<h<90 \mathrm{~km}$, the values for $\log \sigma_{\|}$and $\log \sigma_{P}$ are interpolated by cubic functions of $h$.

The model (Denisenko et al. 2008) permits us to calculate conductivities in the ionosphere only above $h=80 \mathrm{~km}$ since the model IRI is not applicable below this height. These calculations show that all components of the conductivity tensor are defined by electrons below $h=90 \mathrm{~km}$ and all ions give negligible contributions. We suppose that such a domination takes place also in the whole layer $50 \mathrm{~km}<h<90 \mathrm{~km}$ where the values for $\sigma_{\|}$and $\sigma_{P}$ we obtain by continuation of the ionospheric height distributions.

For plasma with one dominating kind of charged particles, the formulae for conductivities written in Hargreaves (1979) are simplified. Then they give the following relation between components of the conductivity tensor

$$
\sigma_{H}(h)=\sqrt{\sigma_{P}(h)\left[\sigma_{\|}(h)-\sigma_{P}(h)\right]} .
$$

So it is not necessary to interpolate the values for $\sigma_{H}$; it can be deduced from this formula after interpolation of $\sigma_{\|}$and $\sigma_{P}$. The Hall parameter $\sigma_{H} / \sigma_{P}$ approximately equals the ratio between the electron gyrofrequency and the electron-neutral collision frequency. As Fig. 7 shows, it takes a value of about 25 at the height $90 \mathrm{~km}$. For $\sigma_{\|}>\sigma_{P}$ the formula (17) means $\sigma_{\|} / \sigma_{H} \simeq \sigma_{H} / \sigma_{P}$. Since such an equality is valid at the height $85-95 \mathrm{~km}$, we can use this approximation. We extrapolate it down to $50 \mathrm{~km}$. In our model, the Hall parameter equals zero below $50 \mathrm{~km}$ which corresponds to isotropic conductivity there.

As we will see later, the details of conductivity profiles in this layer $50 \mathrm{~km}<h<90 \mathrm{~km}$ are of no matter for our model. Only two properties must be represented. The conductivity in the vertical direction is much larger than that in the atmosphere below it and the conductivity in the horizontal direction is much smaller than that in the ionosphere above it. For some other processes, the conductivity in the D-layer is important. For example, Schumann resonances are sensitive to a vertical shift of the profiles which can be by up to $10 \mathrm{~km}$ at these altitudes (Schlegel and Fullekrug 2002).

The typical mid-latitude height distributions are shown in Fig. 7 for night-time conditions in summer under high solar activity. It should be mentioned that these are averaged profiles and the values of actual conductivity on a particular day can be a few times different. The dashed lines in Fig. 7 present the effective Pedersen and Hall conductivities, which describe the ionospheric conductor accelerated by Ampere's force. Such an acceleration would make the conductor move with a drift velocity if the time is long enough and no other force exists. Here we use an averaged acceleration period of $\tau_{A}=3 \mathrm{~h}$. A detailed 
Fig. 7 Profiles of the components of the electric conductivity tensor for a mid-latitude night-time ionosphere. Plotted are the field-aligned conductivity $\sigma$, the Pedersen conductivity $\sigma_{p}$, and the Hall conductivity $\sigma_{H}$ (solid lines). The effective Pedersen and Hall conductivities averaged during an acceleration period of $3 \mathrm{~h}$ are presented by the dashed lines

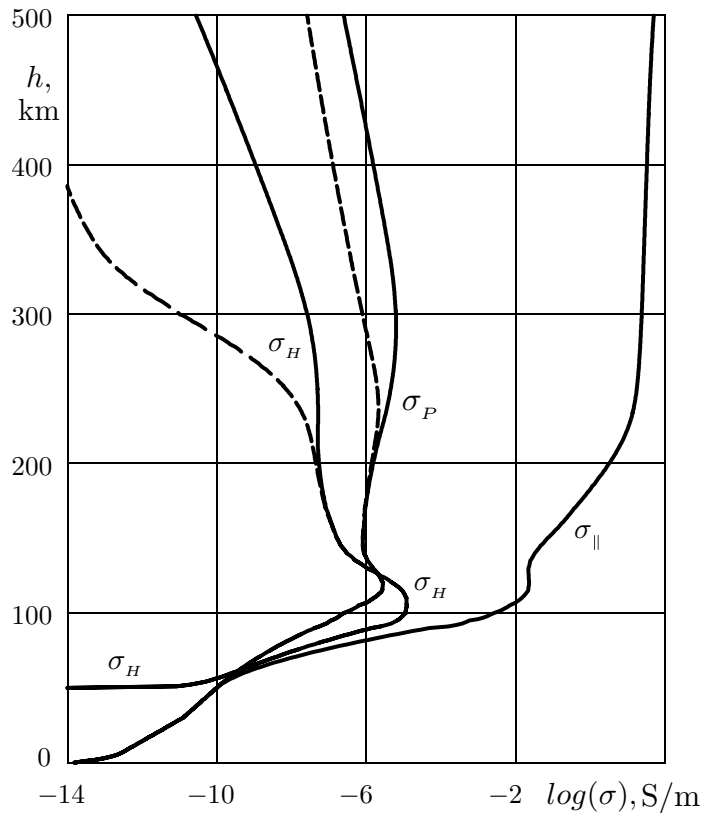

explanation can be found in Denisenko et al. (2008). Sometimes this effect is taken into account in a simplified form as neglecting $\sigma_{P}, \sigma_{H}$ above $160 \mathrm{~km}$ (Forbes 1981). It is not adequate for $\sigma_{P}$ in the night-time ionosphere as can be seen in Fig. 7.

\section{2-D Model of the lonospheric Conductor}

Hargreaves (1979) shows how to reduce a three-dimensional model to a two-dimensional one when the conductivity in the direction of the magnetic field $\sigma$ is a few orders of magnitude larger than $\sigma_{p}, \sigma_{H}$. We follow the approach of Gurevich et al. (1976) where this procedure is made accurately from the mathematical point of view. Our version of this type of model is presented in Denisenko (2018). Here we briefly present only the key features of the model.

As can be seen in Fig. 7, the conductivity in the direction of the magnetic field $\sigma_{\|}$is a few orders of magnitude larger than $\sigma_{P}, \sigma_{H}$ in the layer where $\sigma_{P}, \sigma_{H}$ are large. It is possible to idealize this inequality as

$$
\sigma_{\|}=\infty
$$

in some layer $h_{I}<h<h_{M}$ for which parameters $h_{I}, h_{M}$ are to be chosen.

The equality (18) means that the electric current along a magnetic field line can be arbitrary, while the electric field component $E_{\|}$equals zero,

$$
E_{\|}=0 \text {. }
$$

Because of equations $(4,19)$ the electric potential $V$ is constant at each magnetic field line and

$$
\mathbf{E}_{\perp}=-\operatorname{grad}_{\perp} V .
$$


$\mathbf{a}$

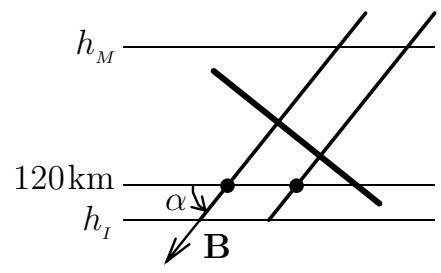

b

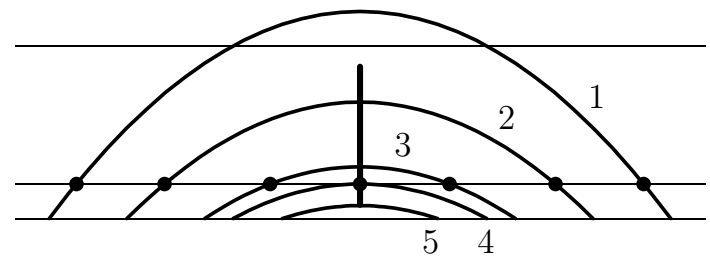

Fig. 8 Magnetic field lines in the ionospheric layer between the heights $h_{I}$ and $h_{M}$ where conductivities $\sigma_{P}$, $\sigma_{H}$ are large. a For middle latitudes, b for equatorial ionosphere. $\alpha$-magnetic inclination. Geomagnetic coordinates of the plotted black dots are used to identify in global pictures the ionospheric parts of the lines which contain these dots. Dark segments indicate cross sections of possible coordinate surfaces which can be used in 2-D models

Two such equipotential segments are shown in Fig. 8 a for middle latitudes. Panel b shows the equatorial ionosphere. A couple of magnetic field lines separate the cross sections of magnetic field tubes which are analyzed below. Since each magnetic field line is an equipotential the ionospheric conductor may be represented by Pedersen and Hall conductances which are equal to integrals of the corresponding local conductivities $\sigma_{P}, \sigma_{H}$ (Hargreaves 1979).

In such a model, a magnetic field line has its own value of the electric potential $V$. It can obtain or lose charge by currents $\mathbf{j}_{\perp}$ and it does not matter for its total charge at what point along the magnetic field line $\mathbf{j}_{\perp}$ exists, because charges can go freely along the line according to infinite $\sigma_{\|}(18)$. Then $\mathbf{E}_{\perp}$ is constant in this integration and so

$$
\mathbf{J}_{\perp}=\left(\begin{array}{cc}
\Sigma_{P} & -\Sigma_{H} \\
\Sigma_{H} & \Sigma_{P}
\end{array}\right) \mathbf{E}_{\perp},
$$

where Pedersen and Hall conductances $\Sigma_{P}, \Sigma_{H}$ are obtained by integration along a magnetic field line

$$
\Sigma_{P}=\int \sigma_{P} \mathrm{~d} l, \quad \Sigma_{H}=\int \sigma_{H} \mathrm{~d} l .
$$

Of course, it is not necessary to integrate along the whole magnetic field line because of small $\sigma_{P}, \sigma_{H}$ outside some layer $h_{I}<h<h_{M}$. As we already wrote $h_{M}=500 \mathrm{~km}$ can be taken as the upper boundary. We use $h_{I}=90 \mathrm{~km}$ because of small conductivity below this height. Calculations show that inclusion of the conductivity outside this layer would increase the integral Pedersen conductivity and day-time Hall conductivity by less than $1 \%$, which is negligible. The night-time Hall conductivity can be increased up to $3 \%$ by the layer below $90 \mathrm{~km}$. We have to accept this error for the reasons described in Sect. 10 . Under high solar activity, the lower boundary of the ionosphere can be shifted about $20 \mathrm{~km}$ down if one defines it as the height with some fixed electron concentration. Nevertheless the ionization increases at all heights and so the relative contribution of the layer below $90 \mathrm{~km}$ does not increase.

Strictly speaking there must be some geometrical factors in the integrals (22) since the neighboring magnetic field lines are not parallel (Denisenko 2018). We divide a magnetic field line into three parts: Northern, magnetospheric and Southern. The magnetospheric part of an equatorial line like the lines 4, 5 in Fig. 8 is absent and the parts are separated by the top. The Northern and Southern segments are embedded in the layer $h_{I}<h<h_{M}$ with nonzero 
conductivities. We approximately regard neighboring segments as parallel ones and take the geometrical factors into account only for the magnetospheric part of the line. It has zero effect on the integrals (22) but defines correspondence of the conjugate points in the hemispheres (Denisenko 2018). So the limits of integration for the Northern and Southern segments in (22) correspond to the points at which the line crosses the lower and upper boundaries of the conducting layer, shown as the height interval $h_{I}<h<h_{M}$ in Fig. 8. For an equatorial magnetic field line, the upper limit corresponds to its top.

The integral conductance $\Sigma_{P}$ or $\Sigma_{H}$ at each half of a magnetic field line can be shown at the surface $h=120 \mathrm{~km}$ in the dot where the line crosses the surface. In other words, a half of a magnetic field line is substituted with a dot as is shown in Fig. 8. It must be mentioned that equatorial magnetic field lines which are below $h=120 \mathrm{~km}$, such as the line 5 in Fig. $8 \mathrm{~b}$, are absent in those pictures. If we choose the height of such a surface below $h=120 \mathrm{~km}$ the dots presenting these lines would appear. Since corresponding values of $\Sigma_{P}, \Sigma_{H}$ are small because of the small length of these lines, it would produce an extra singularity in pictures. We would like to stress that it is a problem of visualization only and does not exist in the calculations.

To calculate the local $\sigma_{P}, \sigma_{H}$ in accordance with Denisenko 2018, to integrate $\Sigma_{P}, \Sigma_{H}$ in the Northern and Southern hemispheres (22) and to trace the magnetic field lines in the magnetosphere we use the IGRF model of the geomagnetic field. This model presents the field that is created by currents which exist inside the Earth as a sum of 65 spherical harmonics with empirically found coefficients. If the geomagnetic field is supposed to be a dipolar one then only the first harmonic is used. The geomagnetic equator would be changed by the straight line $\lambda_{m}=0$ in Fig. 9. The position of the actual geomagnetic equator differs from the dipolar one mainly between Brazil and Africa at $20^{\circ}<\varphi_{m}<100^{\circ}$.

The empirical model IRI does not present any auroral enhancement of electron concentration that is produced by high energy electron and proton precipitation from the magnetosphere. A corresponding enhancement of conductivity is usually added as the auroral zones with large integral conductances $\Sigma_{P}, \Sigma_{H}$. These values are rather variable. We use some average values of the models (Kamide and Matsushita 1979; Spiro et al. 1982; Weimer 1999).

The obtained global distributions of $\Sigma_{P}, \Sigma_{H}$ are presented in Fig. 9; a logarithmic scale is used since the values vary by almost four orders of magnitude. Fig. 10 shows high-latitude fragments of the same $\Sigma_{P}, \Sigma_{H}$. Both Northern and Southern fragments are shown as viewed from the Northern pole.

Figure 9 demonstrates a rather complicated global distribution of conductivity at any fixed moment of time. The main reason for $\Sigma_{P}, \Sigma_{H}$ variations is the solar radiation. We can see small values of $\Sigma_{P}, \Sigma_{H}$ (blue) in night time which may be 2 orders of magnitude less than their daytime values. As we see in Fig. 9, the conductances $\Sigma_{P}, \Sigma_{H}$ are larger in the Northern hemisphere. The Northern polar cap is exposed to the solar radiation because we study a summer. It is the model for 19:00 UT in July. So the local midnight occurs around $\varphi_{m}=145^{\circ}$. The second, clearly seen singularity is the auroral enhancement.

The Pedersen conductivity $\Sigma_{P}>0.2 \mathrm{~S}$ in our model is about $10 \mathrm{~S}$ in middle-latitude daytime ionosphere and increases up to $150 \mathrm{~S}$ near the geomagnetic equator. It is in contrast with the constant value $0.05 \mathrm{~S}$ for the whole ionosphere in the model of Hays and Roble (1979).

The charge conservation law (2) for the 2-D model is satisfied as integrated along a magnetic field line. Using Ohm's law (21) and the expression of the electric field strength by formula (20), we obtain the equation for the electric potential $V$. It is useful to construct some plane with Cartesian coordinates $x, y$ that crosses all magnetic field lines of interest and to write this equation as

$$
-\frac{\partial}{\partial x}\left(\Sigma_{x x} \frac{\partial V}{\partial x}+\Sigma_{x y} \frac{\partial V}{\partial y}\right)-\frac{\partial}{\partial y}\left(\Sigma_{y x} \frac{\partial V}{\partial x}+\Sigma_{y y} \frac{\partial V}{\partial y}\right)=Q_{\text {ext }},
$$



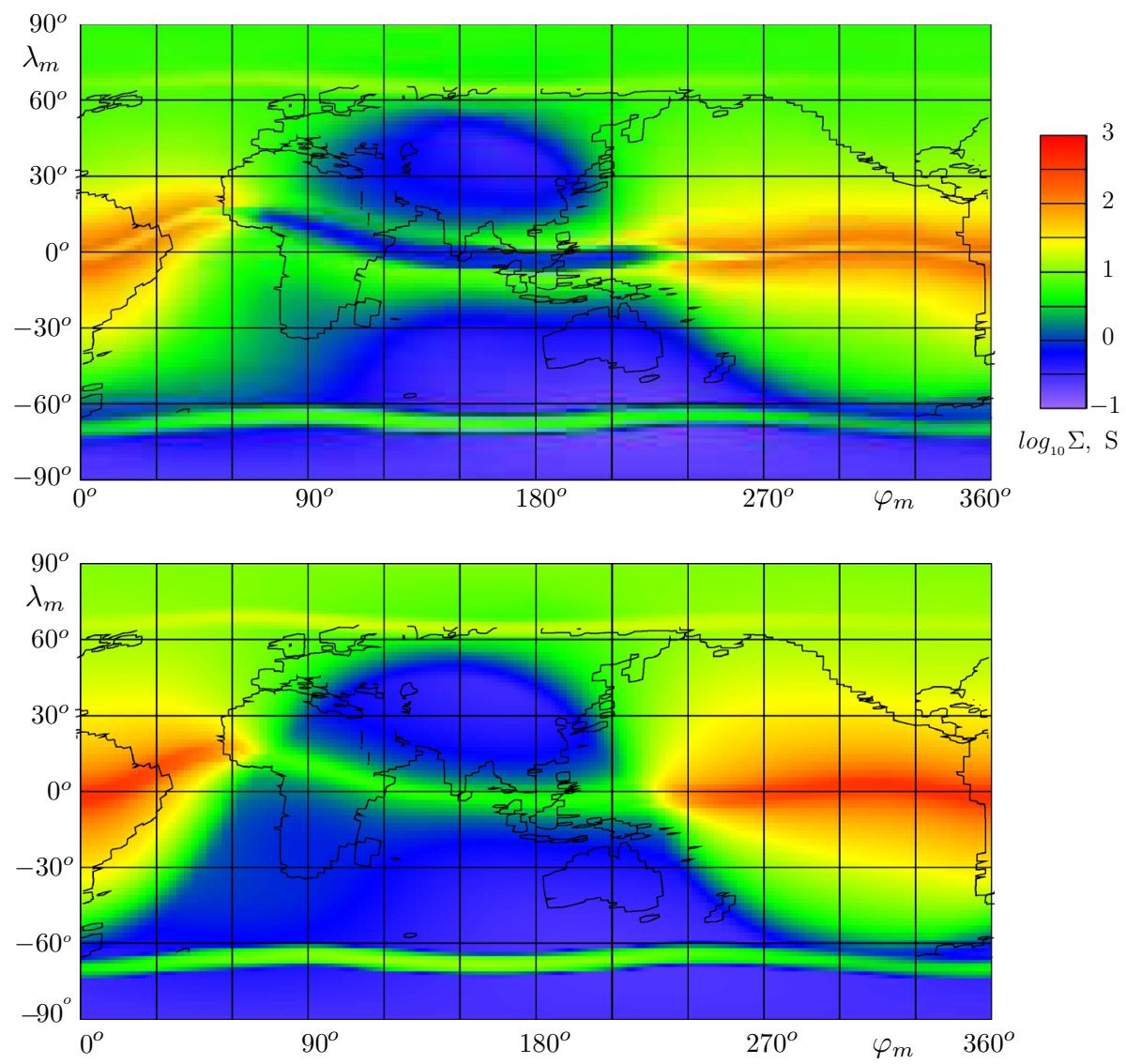

Fig. 9 Distribution of the integral Pedersen conductance $\Sigma_{p}$ (top panel) and Hall conductance $\Sigma_{H}$ (bottom panel). The points with $\lambda_{m}, \varphi_{m}$ geomagnetic coordinates at $120 \mathrm{~km}$ height in the ionosphere identify halves of magnetic field lines. Maps are calculated under typical conditions for July under high solar activity at the considered point in time, 19:00 UT

where $Q_{\text {ext }}$ is the density of current from the atmosphere, that is already found in Sect. 9, transformed to the new coordinates $x, y$. The coefficients $\Sigma_{x x}, \Sigma_{x y}, \Sigma_{y x}, \Sigma_{y y}$ differ from $\Sigma_{p}$, $\Sigma_{H}$ only by geometrical factors defined by the chosen plane. For the main part of the ionosphere, a part of the cross section of such a plane is shown as a dark segment in Fig. 8b.

The partial differential equation (23) is an equation of elliptical type which means that one can use boundary conditions similar to those for Poisson's equation.

\section{Boundary Value Problems}

Because of large $\sigma_{\|}$the magnetospheric conductors are connected with the ionosphere. Our way to take them into account is presented in Denisenko (2018). The magnetic field lines from the auroral zones go to the magnetopause (region 1 in Fig. 11) or to the plasma sheet (region 2 in Fig. 11), where the conductance across magnetic field lines is about $100 \mathrm{~S}$ (Cattell 1996). As the result of a detailed consideration (Denisenko 2018), we can put $V=0$ in 


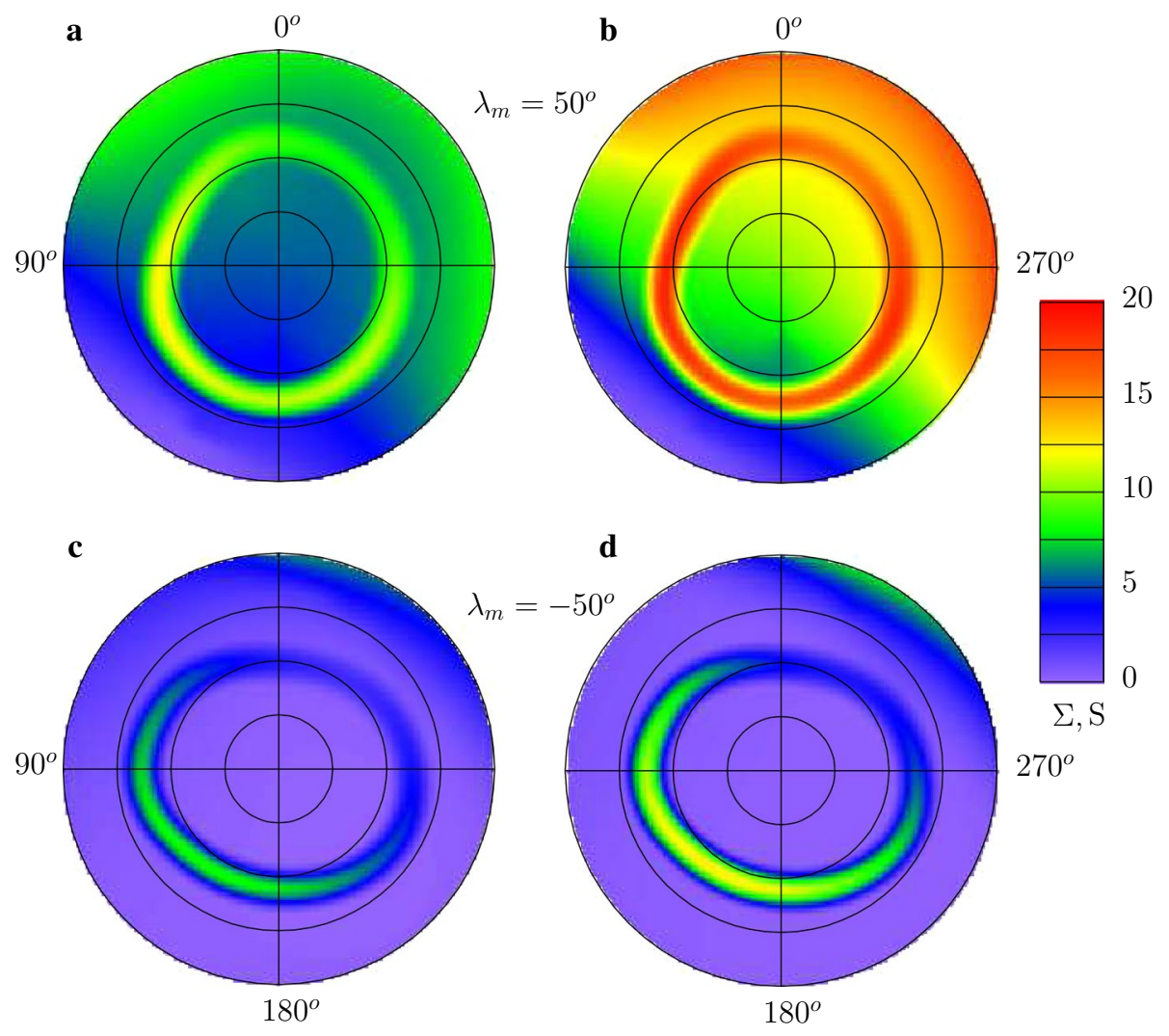

Fig. 10 Distribution of the integral Pedersen $\Sigma_{p}(\mathbf{a}, \mathbf{c})$ and Hall $\Sigma_{H}(\mathbf{b}, \mathbf{d})$ conductances in the Northern $(\mathbf{a}$, b) and Southern $(\mathbf{c}, \mathbf{d})$ polar regions. Maps are calculated under typical conditions for July under high solar activity at the considered point in time, 19:00 UT

Fig. 11 Mapping of the plasma sheet (2) and magnetopause (1) to the ionosphere. (3)-Closed magnetic field lines, (4)_polar cap with open magnetic field lines. Reproduced from Denisenko et al. (2003)

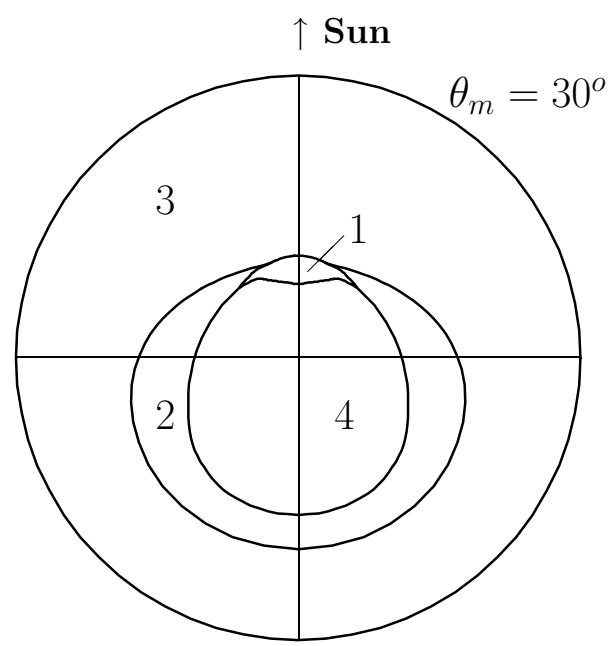


the auroral zones. The auroral zones are equivalent to almost ideal conductors because they are connected in parallel with good magnetospheric conductors. We approximately regard them as ideal ones. The electric potential $V=$ const at an ideal conductor and its value can be taken as zero since $V$-const corresponds to the same electric field as $V$ in view of (4).

This condition cuts the ionosphere into three parts which are the Northern (region 4 in Fig. 11) and Southern polar caps and the main part that contains middle and low latitudes (region 3 in Fig. 11). These parts of the ionospheric conductor in many aspects can be analyzed independently since the potential at their boundaries is already defined as zero:

$$
\begin{gathered}
\left.V\right|_{\Gamma_{\mathrm{N}}}=0, \\
\left.V\right|_{\Gamma_{\mathrm{S}}}=0, \\
\left.V\right|_{\Gamma_{\text {aur }}}=0,
\end{gathered}
$$

where we denote the boundaries of the Northern and Southern polar caps as $\Gamma_{\mathrm{N}}$ and $\Gamma_{\mathrm{S}}$. The auroral boundary of the main part of the ionosphere is $\Gamma_{\text {aur }}$. They are the boundaries of three corresponding flat domains $\Omega_{\mathrm{N}}, \Omega_{\mathrm{S}}$ and $\Omega$ in appropriate Cartesian coordinates. Strictly speaking the positions of these boundaries are not well defined. So we analyze their influence on the results in Sect. 14 and show that it is small.

The interior boundary $\Gamma_{\mathrm{eq}}$ of $\Omega$ corresponds to the last magnetic field lines which are regarded as ionospheric ones and so as equipotential ones. For simplicity and clarity, we first consider the points of this boundary near which the magnetic field has the form shown in Fig. 8 b. A dark segment is a cross section of a part of the domain $\Omega_{N}$ that is near the boundary $\Gamma_{\text {eq }}$. The lower point of this segment belongs to the considered boundary, that is, the magnetic field line 5 is the last one. Above it there is the ionosphere, in which the approximation $\sigma_{\|}=\infty$ (18) is used, which made it possible to construct a 2-D model. The boundary condition as a consequence of the charge conservation law is

$$
\left.J_{v}\right|_{\Gamma_{\text {eq }}}=-J_{\text {eq }}^{0},
$$

where the subscript $v$ denotes the current component normal to the boundary. In view of (21) this boundary condition defines the value of the inclined derivative of $V$.

Such a boundary condition coupled with the large integral conductance near the boundary forms the electrojet whose current is defined by the Cowling conductance $\Sigma_{C}=\Sigma_{P}+\Sigma_{H}^{2} / \Sigma_{P}$ (Forbes 1981). The thin strip near the boundary where the electrojet exists can be separated with a special boundary condition to improve the effectiveness of the numerical method (Denisenko 1998); however, in the actual model we use the original condition (27).

While integral conductances are calculated as described in the previous section, we use the IGRF model of geomagnetic field. This model presents only the field that is created by currents which exist inside the Earth. Such a field is dominant in the ionosphere, but it decreases in the magnetosphere far from the ionosphere, and tracing the magnetic field lines needs consideration of the fields produced by magnetospheric currents. First of all, there are currents at the magnetopause which close the geomagnetic field inside the magnetosphere and currents in the current sheet which pull the magnetic field from the Earth to the tail. It seems to us that the best empirical model of magnetospheric magnetic field is the one created by Tsyganenko and Sitnov (2007). Since we are interested only in closed magnetic field lines starting in the region 3 in Fig. 11, which do not extend too far from the Earth, we use our more simple model (Denisenko et al. 2006) in addition to the field of the 
model IGRF. Tracing of magnetic field lines is necessary to find conjugate points in the Northern and Southern hemispheres since these points have equal potentials because of the high conductivity along a magnetic field line. It means that these conductors in the Northern and Southern hemispheres are connected in parallel and one must take this circumstance into account for the ionospheric electric field and current simulation as we describe in next section.

We are to solve the Dirichlet boundary value problem $(23,24)$ for the unknown function $V$ in 2-D flat domain $\Omega_{N}$ and a similar boundary value problem for the Southern polar cap $(23,25)$. For the main part of the ionosphere, a more complicated boundary value problem $(23,26,27)$ appears in the domain $\Omega$. Each of these problems has a unique solution (Denisenko 1995).

The main difficulties in solving these boundary value problems are related to the Hall conductivity that makes operators of these boundary value problems not symmetrical ones. We proposed new statements with symmetrical positive definite operators which correspond to minimization of the total Joule dissipation. In a particular case $\Sigma_{x x}=\Sigma_{y y}=1$, $\Sigma_{x y}=\Sigma_{y x}=0$, Eq. (23) is the Poisson equation and our variational principle for the problem $(23,24)$ is the Dirichlet principle. The new principles are proven for different 2-D boundary value problems in Denisenko (1994) and Denisenko (2002) and for a 3-D problem in Denisenko (1997). It is simple to apply the finite element method for such a problem. We construct a grid with the usual restrictions and use piece-wise linear functions to approximate the solution. The system of linear algebraic equations for the set of grid values are obtained as the condition of a minimum of the energy functional (total energy). We use a multigrid method to solve these algebraic equations because it is the most effective method for the matrixes which approximate operators of elliptical type. Our numerical method for such a problem is described in detail in Denisenko (1998), including a new statement of the boundary value problem, the finite element method, the multigrid method, and some test calculations. Test calculations which use different grids show that the relative error is much less than $1 \%$ and so the errors of calculations are negligible. Of course, the precision of the model is much worse because of poor definition of the input parameters.

\section{The Results of the Calculations}

We solve numerically three independent boundary value problems. They are Dirichlet boundary value problems $(23,24),(23,25)$ in the polar caps and the boundary value problem of mixed type $(23,26,27)$ in the main part of the ionosphere. Here we study electric potential variations inside the ionosphere. Because of the high ionospheric conductivity in comparison with atmospheric conductivity, these variations are small in comparison with the $200-300 \mathrm{kV}$ voltage between the ionosphere and ground.

The solution for the boundary value problem $(23,26,27)$ in the main part of the ionosphere is presented in Fig. 12. The distribution of the electric potential $V\left(\theta_{m}, \varphi_{m}\right)$ at height $h=120 \mathrm{~km}$ in the ionosphere is shown by the positions of the equipotentials, which are plotted with a contour interval equal to $2 \mathrm{~V}$. Figure 13 similarly shows the solutions for the problems $(23,24)$ and $(23,25)$ in the polar caps, the contour interval equals $0.1 \mathrm{~V}$ in the Northern cap and $5 \mathrm{~V}$ in the Southern cap. Maximum potential differences are about $0.7 \mathrm{~V}$ in the Northern cap, $22 \mathrm{~V}$ in the Southern cap and $42 \mathrm{~V}$ in the main part of the ionosphere. Such a great difference between the polar caps appears because the Southern cap is in darkness in July and so the conductivity of its ionosphere is only 


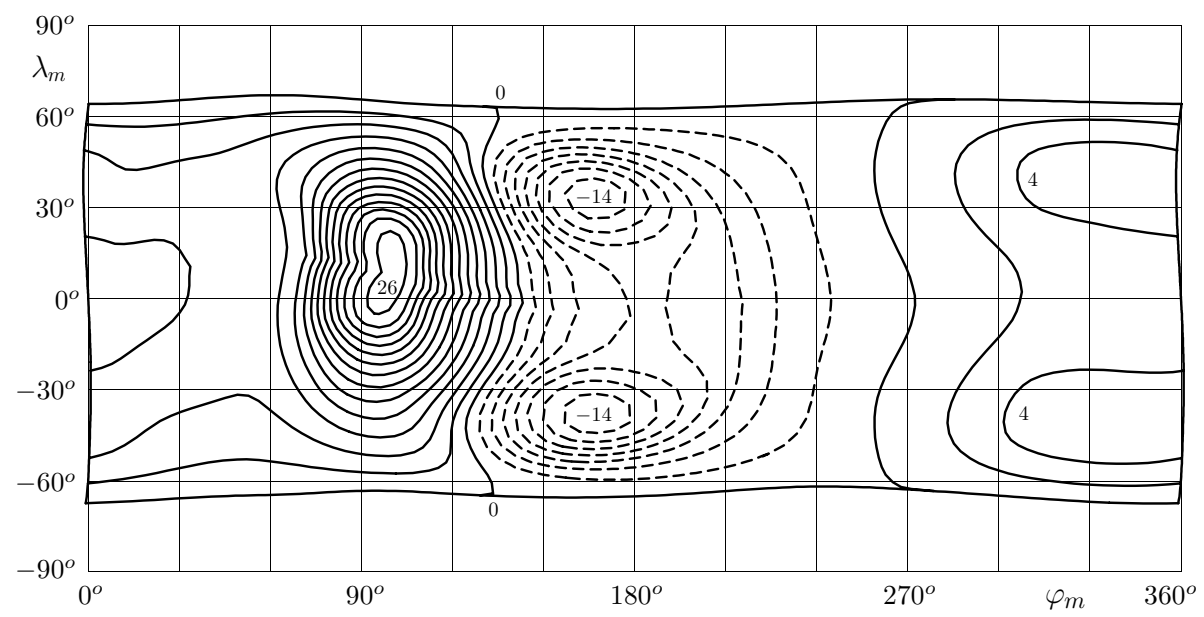

Fig. 12 Distribution of the electric potential at $120 \mathrm{~km}$ height in the ionosphere. Equipotentials are plotted with contour interval $2 \mathrm{~V}$. Dashed lines correspond to negative values of potential. Map is calculated under typical conditions for July under high solar activity at the considered point in time, 19:00 UT
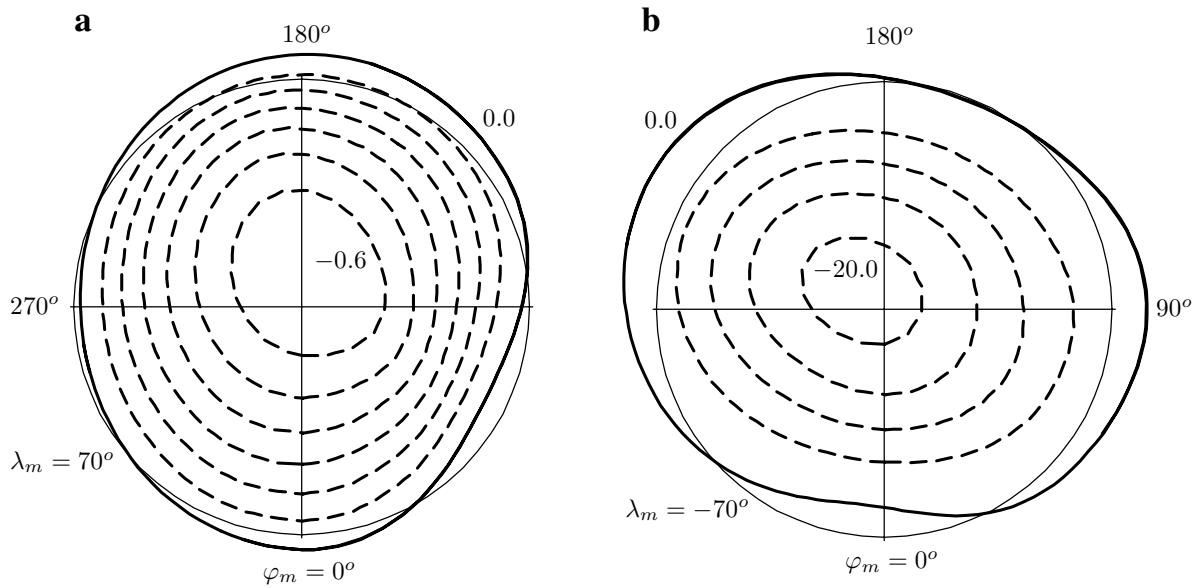

Fig. 13 Distribution of the electric potential at $120 \mathrm{~km}$ height in the polar caps. Contour interval equals $0.1 \mathrm{~V}$ in the Northern cap (a) and $5 \mathrm{~V}$ in the Southern cap (b). Dashed lines show negative values

a few percent of the conductivity in the Northern cap, as is shown in Fig. 10. There is no chance to measure these small voltages since hundreds of times larger voltages of other nature exist in the low latitude ionosphere (Fejer 1981; Forbes 1981), stronger ones in middle latitudes and up to $100 \mathrm{kV}$ in high latitudes (Kamide et al. 1981).

The obtained distribution of the electric potential $V$ shown in Fig. 12 permits us to calculate the electric field strength by formula (4). Its horizontal components are shown in Fig. 14. Instead of $\lambda$ - and $\varphi$-components, we prefer to use $E_{1}$ directed along the horizontal part of the vector $\mathbf{B}$ and $E_{2}$ normal to $E_{1}$. These directions better fit to the magnetic field in low latitudes. In particular just $E_{1}=0$ at the geomagnetic equator because 

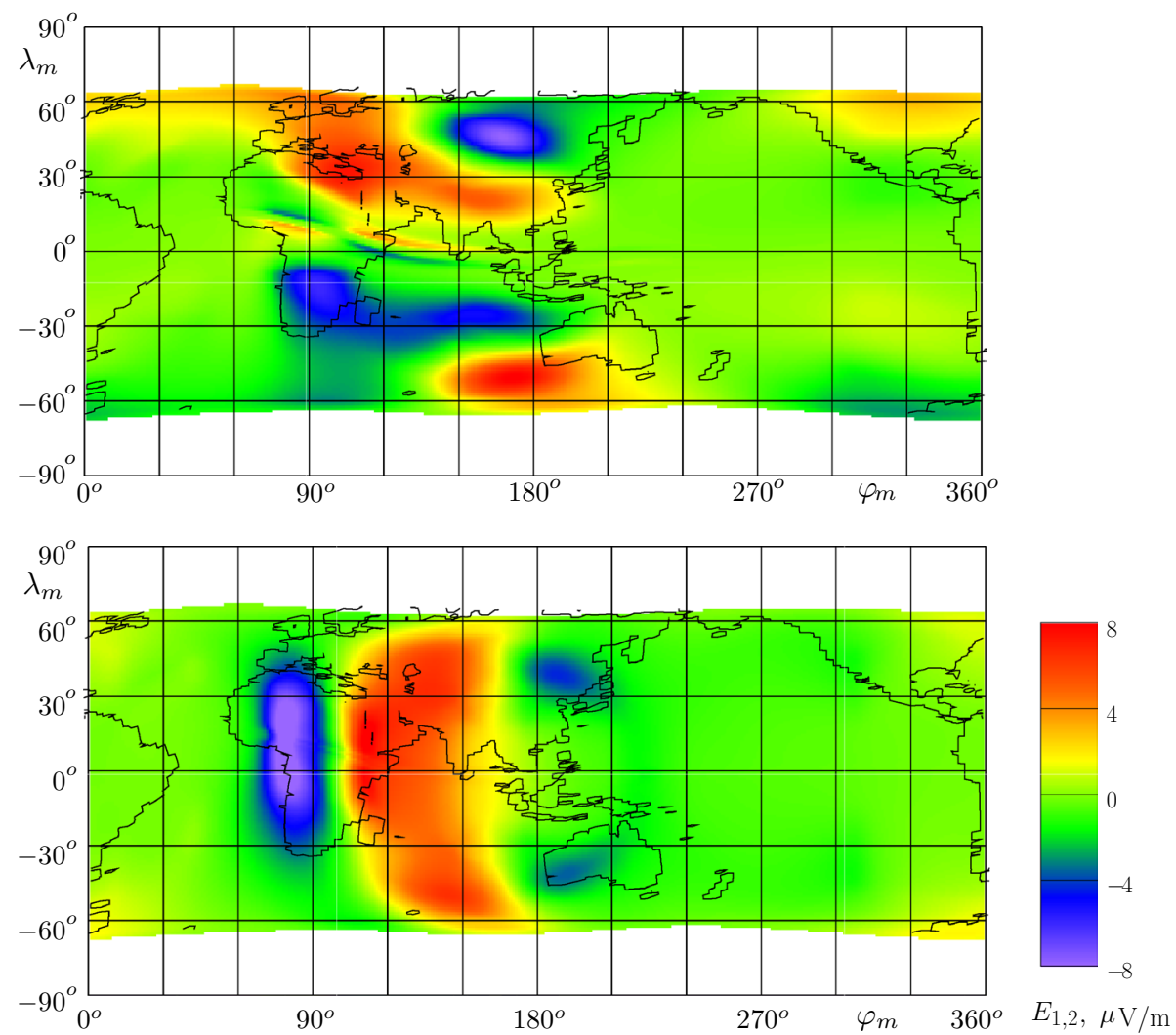

Fig. 14 Distributions of the horizontal components of the electric field strength $E_{1}$ (top panel) and $E_{2}$ (bottom panel) which are close to $E_{\lambda}$ and $E_{\varphi}$, respectively. This $\mathbf{E}$ corresponds to the potential shown in Fig. 12

it is parallel to the vector $\mathbf{B}$ there and so $E_{1}=E_{\|}=0$ (19). If the geomagnetic field is a dipolar one then $E_{1}=E_{\lambda}, E_{2}=E_{\varphi}$.

The field-aligned component of the electric field in the ionosphere is small because of huge field-aligned conductivity $\sigma_{\|}$and equals zero in our model (19). It means that only normal to the magnetic field components of $\mathbf{E}$ exist. They are approximately horizontal in high latitudes and so the vertical component of $\mathbf{E}$ is large only in the low latitudes. Therefore, it is useful to plot one more picture additionally to Fig. 14 to present the whole vector $\mathbf{E}$.

The absolute value of the electric field strength $|\mathbf{E}|$ is presented in Fig. 15. We see relatively large $|\mathbf{E}|$ in the equatorial ionosphere. It is mainly a vertical component of the electric field since Fig. 14 shows no amplification of the horizontal components there in comparison with middle latitudes. Maximum $|\mathbf{E}|$ at $120 \mathrm{~km}$ height is about $75 \mu \mathrm{V} / \mathrm{m}$ and it is reached within the equatorial singularity. This singularity is similar to the singularity in the equatorial electrojet (Forbes 1981). Almost everywhere in middle latitudes $|\mathbf{E}| \leq 10 \mu \mathrm{V} / \mathrm{m}$ and $|\mathbf{E}| \leq 1 \mu \mathrm{V} / \mathrm{m}$ in the main part of the day-time ionosphere. It is much less than electric fields of other natures which usually are in the $\mathrm{mV} / \mathrm{m}$ range in the low latitude ionosphere and larger in middle and especially in high latitudes. 


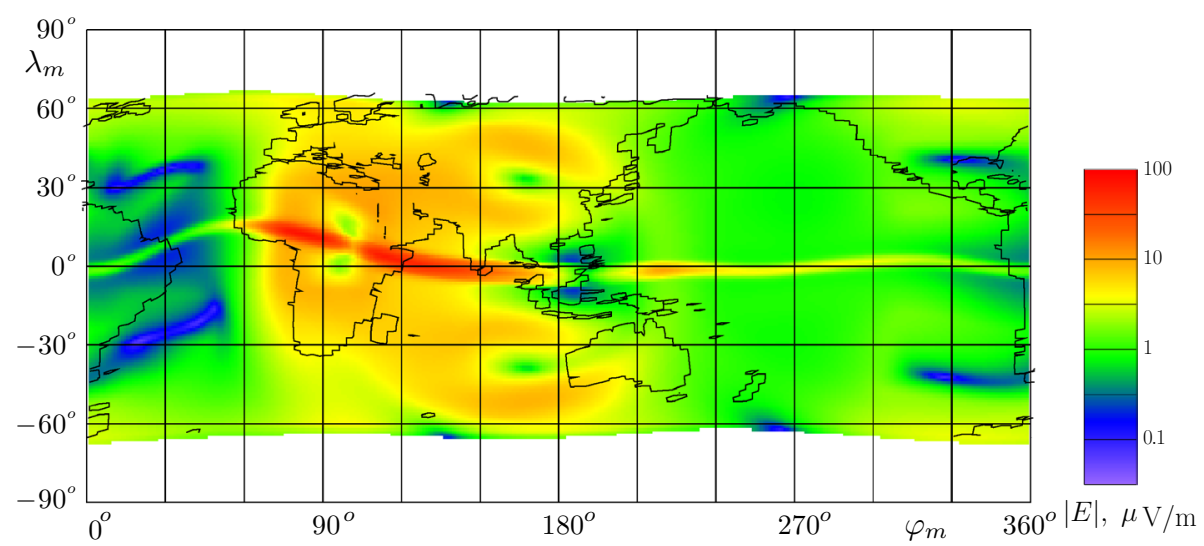

Fig. 15 Distribution of magnitude of the electric field strength $|\mathbf{E}|$ at $120 \mathrm{~km}$ height corresponding to the potential shown in Fig. 12

As can be seen in Fig. 12 the horizontal part of the electric field near the geomagnetic equator is directed almost along the equator; that is the $E_{2}$ component shown in Fig. 14, $\left|E_{2}\right| \leq 9 \mu \mathrm{V} / \mathrm{m}$ in our model. These values do not agree with the results of the model (Kartalev et al. 2006) that gives an order of magnitude larger equatorial electric field in the ionosphere around similar thunderstorm region in Africa. The difference can be because we simulate the ionosphere at 19:00 UT when Africa is near the terminator in contrast with the model (Kartalev et al. 2006) designed for midnight in Africa when the ionospheric conductivity is much smaller as is shown in Fig. 9 and so the same currents are provided with much stronger electric fields.

\section{Discussion of the Numerical Results}

The key input parameter of our model is the space distribution of the ionospheric conductivity. Here we use its effective values decreased due the ionospheric conductor acceleration by Ampere's force with a typical period of $\tau=3 \mathrm{~h}$ (Denisenko et al. 2008) as is described in Sect. 8. The value of $3 \mathrm{~h}$ is not a well-defined parameter. Sometimes this effect is taken into account in a simplified form as neglecting $\sigma_{P}, \sigma_{H}$ above $160 \mathrm{~km}$ (Forbes 1981) that means $\tau_{A}=\infty$. During $3 \mathrm{~h}$ the Earth rotates $45^{\circ}$ and so the ionospheric medium at any point for sure is subjected to another electric field strength. So $\tau_{A}>3 \mathrm{~h}$ has little physical sense. This effect can be studied quantitatively only in the frame of much more complicated models of the ionosphere which include simulation of the motion of the medium such as the ionospheric parts of the models (Kuo et al. 2014; Namgaladze et al. 2013).

The original values of $\sigma_{P}, \sigma_{H}$ are much larger above $200 \mathrm{~km}$ as is seen in Fig. 7. This effect is important for the night-time ionosphere since its integral conductances $\Sigma_{P}, \Sigma_{H}$ are rather small below this height. If we use not these effective but the original values, the ionospheric integral conductances $\Sigma_{P}, \Sigma_{H}$ would be a few times larger, because they would be equal to the integrals of $\sigma_{P}, \sigma_{H}$ shown by the solid lines in Fig. 7. Since the electric current generators are taken as given in our model, the electric fields would be significantly decreased. As additional calculations show, the maximum voltage in the main part of the 
ionosphere would be $20 \mathrm{~V}$, instead of $42 \mathrm{~V}$ shown in Fig. 12, and about 1.8 times less in the polar caps in comparison with Fig. 13.

In the well-known model (Hays and Roble 1979), the ionospheric integral conductances $\Sigma_{H}=0$ and $\Sigma_{P}$ are decreased much by neglecting with the local conductivity $\sigma_{P}$ above $105 \mathrm{~km}$. Also a constant value was used because of restrictions of their mathematical methods. Hays and Roble (1979) set $\Sigma_{P} \equiv 0.05 \mathrm{~S}$ on purpose to have maximum electric field values and mentioned that the electric field would be smaller if the value of $\Sigma_{p}$ is increased.

Hays and Roble (1979) in Fig. 5a present potential at the height $105 \mathrm{~km}$ in contrast with $h=120 \mathrm{~km}$ in our Fig. 12. A magnetic field line which starts $10^{\circ}$ from the equator shifts to about $50 \mathrm{~km}$ in direction to the equator while it rises from 105 to $120 \mathrm{~km}$. This shift is smaller at larger latitudes and cannot be seen neither in our Fig. 12 nor in Fig. 5a in Hays and Roble (1979). Within the strip $\pm 10^{\circ}$, no potential difference across this strip is shown in Fig. 5a in Hays and Roble (1979) and so the potential value stays the same at $120 \mathrm{~km}$ within the strip $\pm 9.5^{\circ}$ from the equator since each magnetic field line is equipotential in the model. So we can directly compare Fig. 12 and Fig. 5a in Hays and Roble (1979).

Here we use much more realistic distributions of the ionospheric conductance $\Sigma_{P}$ whose values are everywhere larger than $0.17 \mathrm{~S}$ and much larger in the day-time ionosphere because the solar ultraviolet produces ionization. This is shown in Figs. 9 and 10. The Hall conductance $\Sigma_{H}$ that was neglected in the model (Hays and Roble 1979) also decreases the electric field as a rule. It is important especially near the geomagnetic equator where both $\Sigma_{P}$ and $\Sigma_{H}$ define the high Cowling conductance $\Sigma_{C}=\Sigma_{P}+\Sigma_{H}^{2} / \Sigma_{P}$. It is shown in Denisenko et al. (2008) that just $\Sigma_{C}$ is the key parameter of conductivity near the equator but not the integral of the local Cowling conductivity $\sigma_{C}=\sigma_{P}+\sigma_{H}^{2} / \sigma_{P}$. The values of $\Sigma_{C}$ reach $10^{4} \mathrm{~S}$ on magnetic field lines for which each apex is at heights $100-110 \mathrm{~km}$ in the day-time ionosphere. It defines the concentration of the ionospheric currents into equatorial electrojets and simultaneously decreases the longitudinal electric field. Of course, there is no equatorial singularity in the potential distributions in the model (Hays and Roble 1979) because of the simplifications.

One more reason for a lesser voltage in our model compared with the model of Hays and Roble (1979) is the existence of the auroral zones connected to parts of the magnetosphere with high conductivity across magnetic field lines, which are not taken into account in the model (Hays and Roble 1979). These magnetospheric conductors together with the auroral enhancement of conductivity define the electric field in the high-latitude ionosphere, but do not have a large influence on the voltage in the middle latitudes. For example, the maximum voltage would be increased by only $0.5 \%$ from its value $42 \mathrm{~V}$ shown in Fig. 12, if we shift these highly conductive belts $2.5^{\circ}$ poleward. This corresponds approximately to the imprecision of their positions inside the auroral zones and has a negligible influence on the results of the model.

All these features of our model increase the conductance in comparison with the model of Hays and Roble (1979). So it is natural that the electric field strength in our model is much smaller in spite of being almost the same physical model. The model potential distribution at the height $h=105 \mathrm{~km}$ is presented in Fig. 5 (a) in Hays and Roble (1979), and the values of the potential do not vary along magnetic field lines above this height in their model. The maximum potential difference within the ionosphere equals $1575 \mathrm{~V}$. $^{1}$

\footnotetext{
1 By the way there is a misprint in that figure caption. There must be a $100 \mathrm{~V}$ contour interval instead of $1000 \mathrm{~V}$ to fit the text.
} 
This voltage $1575 \mathrm{~V}$ exceeds our $42 \mathrm{~V}$ by about 40 times because of too much decreased conductivities in the model (Hays and Roble 1979) in comparison with conductivities in our model which are set by empirical data.

Nevertheless the positions of the maximum values of the ionospheric potential in the model (Hays and Roble 1979) are close to ours because they correspond to large thunderstorm generators in the center of Africa and in Central America which are dominating as is seen in Fig. 6. The value of the potential above central Africa $V=27 \mathrm{~V}$ in our model is about five times larger than $V=5 \mathrm{~V}$ above Central America as is seen in Fig. 12 in spite of the fact that the current from atmosphere is smaller $(700 \mathrm{~A}$ above central Africa and 420 A above Central America). It happens because of the small ionospheric conductivity in the evening-time ionosphere around $20 \mathrm{LT}$ in comparison with the day-time one around 13 LT as is shown in Fig. 9.

The resulting electric field strength $|\mathbf{E}|$ calculated by formula (4) is presented in Fig. 15. Because of $E_{\|}=0$ (19) in the ionosphere $|\mathbf{E}|=\left|\mathbf{E}_{\perp}\right|$. In high latitudes $\mathbf{E}_{\perp}$ has two horizontal components shown in Fig. 14. Near the geomagnetic equator one component of $\mathbf{E}_{\perp}$ is vertical. The maximum $|\mathbf{E}|=80 \mu \mathrm{V} / \mathrm{m}$ that can be seen in Fig. 15 near the night-time geomagnetic equator mainly presents a vertical component of $\mathbf{E}$ inside the equatorial singularity since $E_{1} \simeq E_{\|}=0$ there and $E_{2} \leq 10 \mu \mathrm{V} / \mathrm{m}$ everywhere as we mentioned above.

Figure 6 (c) in Hays and Roble (1979) presents the longitudinal component of the electric field up to $250 \mu \mathrm{V} / \mathrm{m}$ in that model. It is an order of magnitude larger than the similar component of the electric field $E_{2} \leq 10 \mu \mathrm{V} / \mathrm{m}$ in our model as is shown in Fig. 14 .

It ought to be mentioned that the electric field strength at other heights in the ionosphere outside the equatorial singularity almost does not differ because magnetic field lines are almost parallel at distances less or about hundred km. So the points in Fig. 15 only would be shifted in the direction normal to the geomagnetic equator, from the equator if the height $h$ is decreased in comparison with $120 \mathrm{~km}$ or to the equator if $h$ is increased. The distance of this horizontal shift is negligible in high latitudes and rather small in the main part of the ionosphere. Figure 8a shows that such a shift to the right equals to $(h-120 \mathrm{~km}) / \tan (\alpha)$ where $\alpha$ is the magnetic inclination. The equatorial ionosphere differs much in this respect since additional magnetic field lines appear if we go down. Figure $8 \mathrm{~b}$ shows such an additional line 5 that does not exist at $h=120 \mathrm{~km}$. These equatorial magnetic field lines are absent in Fig. 15. We already mentioned that it is a problem of visualization only and that these lines are taken into account in the calculations.

The total value of the fair weather currents from the ionosphere to ground under steadystate conditions is the same as the total current of the thunderstorm generators, but its distribution over the globe depends much on the atmospheric conductivity. This equality is provided by the voltage between the ionosphere and ground $V_{0}$ whose value was chosen in Sect. 9 for this purpose (14). The vertical atmospheric current density is positive (upward) in the rectangles in Fig. 6, and negative (downward) density outside the rectangles. The downward current density is proportional to the vertical atmospheric conductance shown in the same Fig. 6.

One of the main input parameters of any model of the GEC is the atmospheric conductivity. We use a simple model of height profiles of the atmospheric conductivity that presents only the difference between ground and sea surface and height of ground above sea. The column conductance $\Sigma(\theta, \varphi)(12)$ in such a model varies from about $10^{-17} \mathrm{~S} / \mathrm{m}^{2}$ above sea and above the main part of ground and up to $3 \times 10^{-17} \mathrm{~S} / \mathrm{m}^{2}$ above high mountains as is shown in Figs. 5 and 6. In the paper (Kudintseva et al. 2016) a hybrid profile is constructed. Conductivity is the same as our model below $10 \mathrm{~km}$ and increased about 1.7 


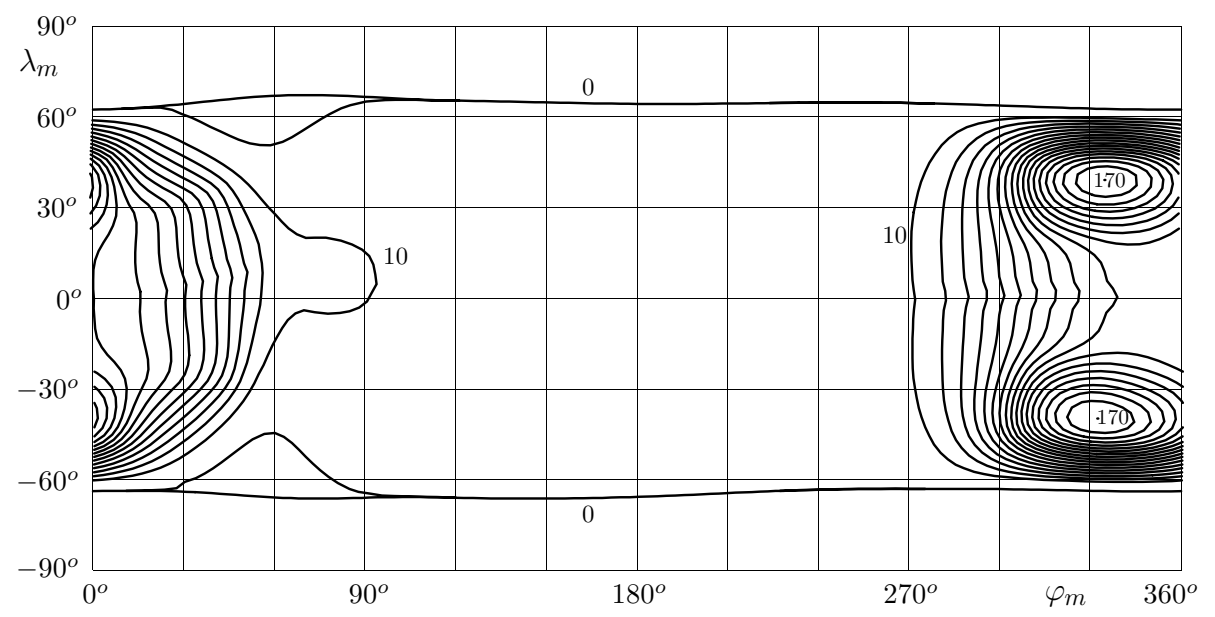

Fig. 16 Distribution of the electric potential at $120 \mathrm{~km}$ height in the ionosphere at 06 : 00 UT, when midnight comes in the Central American thunderstorm area in July of a year of minimal solar activity. Equipotentials are plotted with contour interval $10 \mathrm{~V}$

times at $h=20 \mathrm{~km}$. As is seen in Fig. 5, the air above $20 \mathrm{~km}$ has negligible effects on the conductance of the atmospheric vertical column.

The column conductance of the atmosphere can be varied much because of different reasons. Dust clouds and radon emanation are analyzed in Harrison et al. (2010) and Harrison et al. (2014). One more important reduction in conductivity is made by non-electric clouds. It can be by up to ten times inside clouds (Rycroft and Odzimek 2010; Baumgaertner et al. 2014) but this is a poorly known quantity. The model (Baumgaertner et al. 2014) gives up to twice larger column resistance, mainly above oceans in low latitudes. As a result, the total conductance of the atmosphere would be decreased and the voltage between ionosphere and ground $V_{0}$ would be proportionally increased. Fair weather electric currents would be partially redistributed from low to high latitudes as a result of such a modification of conductivity. So the ionospheric electric fields also would be varied, but their main features are related with thunderstorm areas as we see in Figs. 12 and 16.

At another moment of UT, the ionospheric electric fields may be rather different from those presented in Fig. 15, because the main thunderstorm areas may be under a day-time ionosphere with large conductivity or under a night-time one. In the last case, a larger ionosphere electric field is required to close the currents from the atmosphere. To study this effect, we conducted calculations for 06:00 UT, when midnight comes in the Central American thunderous area. The voltage in the ionosphere increases up to $72 \mathrm{~V}$ in comparison with $42 \mathrm{~V}$ in Fig. 12.

The ionospheric electric fields also vary with solar activity. For 06:00 UT, additional calculations were done for a year of minimal solar activity, namely for Covington index $F 10.7=80$ instead of 190 in our calculations discussed above. The global distributions of $\Sigma_{P}, \Sigma_{H}$ for this case are presented in Denisenko (2018). Their night-time values are almost twice smaller than ones presented in Fig. 9. The obtained distribution of the electric potential $V\left(\theta_{m}, \varphi_{m}\right)$ at height $h=120 \mathrm{~km}$ in the ionosphere is shown in Fig. 16. Negative values are not seen since $V>-8 \mathrm{~V}$ that is less than the contour interval $10 \mathrm{~V}$. The voltage is increased up to $188 \mathrm{~V}$. The maximum value of the ionospheric potential $V=180 \mathrm{~V}$ is 
found above Central America and its conjugate point. The electric field is redistributed and its strength is about four times as strong as the field at 19:00 UT in July of a year of maximal solar activity shown in Fig. 15. Since electric field strength is inversely proportional to the distance between equipotentials, Fig. 16 shows the region $300^{\circ}<\varphi_{m}<370^{\circ}$, $45^{\circ}<\lambda_{m}<60^{\circ}$ around midnight with strong horizontal components of electric field $40 \mu \mathrm{V} / \mathrm{m}<E_{\lambda}<100 \mu \mathrm{V} / \mathrm{m}$. Of course, the same field exists in the conjugate region in the Southern hemisphere. We cannot find such conditions when horizontal components of electric field significantly exceed $100 \mu \mathrm{V} / \mathrm{m}$ even in a small region of the ionosphere for the same atmospheric generators.

\section{Conclusions}

Thus, the goal is achieved: we constructed a mathematical model of the ionospheric electric fields, providing a closure of the currents from the atmosphere into the ionosphere, generated by thunderstorms, and fair weather currents. We found a significant dependence of such electric fields on the Universal Time and on the solar activity. The created model is compared with the well-known model of Roble and Hays (1979). The results are significantly different, since we use a much more sophisticated model of the global ionospheric conductor.

In addition, we have improved the description of the fair weather currents by taking into account the relief and differences between the values of the air conductivity above land and sea.

The key parameters in our model of the GEC are the following: voltage between the ground and the ionosphere $V_{0}=250 \mathrm{kV}$, fair weather electric field near ground $E_{0}=130 \mathrm{~V} / \mathrm{m}$ with corresponding current density $j_{0}=2 \mathrm{pA} / \mathrm{m}^{2}$, the total current from thundery areas to the ionosphere $I_{\text {ext }}=1.4 \mathrm{kA}$. These values are considered to be typical for the GEC, but in view of the linearity of the model they could be multiplied by any common constant. It may be a multiplication by 1.4 to make $I_{\text {ext }}=2 \mathrm{kA}$ and as a consequence $V_{0}$ would be $350 \mathrm{kV}$. The total resistance of the atmosphere in our model $R=180 \Omega$ also could be modified, for example it can be increased by taking clouds into account.

The main result of this research is that the global thunderstorm activity generates a small electric field in the ionosphere. Almost everywhere in middle latitudes $|\mathbf{E}| \leq 10 \mu \mathrm{V} / \mathrm{m}$ and $|\mathbf{E}| \leq 1 \mu \mathrm{V} / \mathrm{m}$ in the main part of the day-time ionosphere. It seems that such a small electric field could not be detected experimentally, at the present time. It seems unlikely that it could ever be observed, but some special circumstances may arise.

The potential difference between ionospheric regions above thundestorm areas and above fair weather areas is always less than $200 \mathrm{~V}$. For the particular time of 19:00 UT in July that is mainly studied in our paper the maximum potential difference inside the E-layer of the ionosphere is $42 \mathrm{~V}$. The maximum of the potential occurs above the African thunderstorm area and the minimum is found above the Himalayas. The potential difference increases up to $72 \mathrm{~V}$ at 06:00 UT, when midnight comes in the Central American thunderstorm area. These thunderstorms generate the strongest current to the ionosphere $(700 \mathrm{~A}$ in our model) and the small night-time ionospheric conductivity needs a relatively strong electric field to spread this current to fair weather regions where it goes down to ground. During a year of minimal solar activity the voltage inside the ionosphere is increased up to $188 \mathrm{~V}$ at 06:00 UT as is shown in Fig. 16. 
This voltage $<200 \mathrm{~V}$ inside the ionosphere is a thousand times less than the voltage between the ionosphere and ground $V_{0}=250 \mathrm{kV}$. Such a relation confirms the possibility to simulate the ionosphere as an ideal conductor while calculating atmospheric electric fields and currents as we did in Sect. 4. Even under minimal solar activity, the horizontal components of the electric field can exceed $0.05 \mathrm{mV} / \mathrm{m}$ only in small regions of the middle-latitude ionosphere and stay less than $0.01 \mathrm{mV} / \mathrm{m}$ in low latitudes. It is of the order of a hundred times less than the strength of other natural electric fields which are in the $\mathrm{mV} / \mathrm{m}$ range even in the low latitude ionosphere (Fejer 1981; Forbes 1981) and much stronger in middle and high latitudes (Kamide et al. 1981).

The obtained value of the maximum horizontal electric field strength in the ionosphere is an order of magnitude less than that in the well-known model of Hays and Roble (1979) that is constructed for the same time, 19:00 UT, in July. Our voltages in the ionosphere are forty or twenty times less for years of maximal or minimal solar activity, respectively. The main reason for the different orders of magnitudes is a better approach for the simulation of the ionospheric conductivity in our model. That is our principal improvement to the model of Hays and Roble (1979).

Funding The research is supported by Russian Foundation for Basic Research (Project 18-05-00195)

Open Access This article is distributed under the terms of the Creative Commons Attribution 4.0 International License (http://creativecommons.org/licenses/by/4.0/), which permits unrestricted use, distribution, and reproduction in any medium, provided you give appropriate credit to the original author(s) and the source, provide a link to the Creative Commons license, and indicate if changes were made.

\section{References}

Ampferer M, Denisenko VV, Hausleitner W, Krauss S, Stangl G, Boudjada MY, Biernat HK (2010) Decrease of the electric field penetration into the ionosphere due to low conductivity at the near ground atmospheric layer. Ann Geophys 28:779-787. https://doi.org/10.5194/angeo-28-779-2010

Aplin KL, Harrison RG, Rycroft MJ (2008) Investigating Earth's atmospheric electricity: a role model for planetary studies. Space Sci Rev 137:11-27. https://doi.org/10.1007/s11214-008-9372-X

Baumgaertner AJG, Thayer JP, Neely RR III, Lucas G (2013) Toward a comprehensive global electric circuit model: atmospheric conductivity and its variability in CESM1(WACCM) model simulations. J Geophys Res 118:9221-9232. https://doi.org/10.1002/jgrd.50725

Baumgaertner AJG, Lucas GM, Thayer JP, Mallios SA (2014) On the role of non-electric clouds in the global electric circuit. Atmos Chem Phys Discuss 14:9815-9847. https://doi.org/10.5194/ acpd-14-9815-2014

Bayona V, Flyer N, Lucas GM, Baumgaertner AJG (2015) A 3-D RBF-FD solver for modeling the atmospheric global electric circuit with topography (GEC-RBFFD v1.0). Geosci Model Dev 8:3007-3020. https://doi.org/10.5194/gmd-8-3007-2015

Cattell CA (1996) Experimental evaluation of the Lundquist number for the Earth's magnetopause and magnetotail. J Geophys Res 101:27309-27316. https://doi.org/10.1029/96JA02448

Denisenko VV (1994) A boundary value problem for an elliptic equation in two variables with asymmetric tensor coefficients. Sib Math J 35(3):495-505. https://doi.org/10.1007/BF02104813

Denisenko VV (1995) Energy methods for elliptic equations with asymmetric coefficients. Publ. House of the Russian Academy of Sciences Siberian Branch, Novosibirsk (in Russian)

Denisenko VV (1997) The energy method for three dimensional elliptical equations with asymmetric tensor coefficients. Sib Math J 38(6):1099-1111. https://doi.org/10.1007/BF02675937

Denisenko VV (1998) Multigrid method for a global Hall conductor in the Earth's ionosphere. In: Virtual Proceedings of the 10-th Anniversary International GAMM-Workshop on Multigrid Methods. http:// www.mgnet.org/mgnet-parmgm98.html

Denisenko VV (2002) A boundary value problem for an elliptic equations with asymmetric coefficients in a non-schlicht domain. Sib Math J 43(6):1055-1068. https://doi.org/10.1023/A:1021169318012 
Denisenko VV (2014a) Electric current penetration from a thunderstorm cloud into the ionosphere at the geomagnetic equator. In: Troyan VN et al (ed) Proceedings of the 10-th international conference "problems of geocosmos". St. Petersburg, pp 82-87

Denisenko VV (2014b) Electric current penetration from a thunderstorm cloud into the middle-latitude ionosphere. In: Troyan VN et al (ed) Proceedings of the 10-th international conference "problems of geocosmos". St. Petersburg, pp 76-81

Denisenko VV (2015) Estimate for the strength of the electric field penetrating from the Earth's surface to the ionosphere. Russ J Phys Chem B 9(5):789-795. https://doi.org/10.1134/S199079311505019X

Denisenko VV (2018) 2-D model of the global ionospheric conductor connected with the magnetospheric conductors. p 15. arXiv.1802.07955

Denisenko VV, Yakubailik OE (2015) The income of topography to the resistance of the global atmospheric conductor. Solar Terr Phys 1(1):104-108. https://doi.org/10.12737/6044 (in Russian)

Denisenko VV, Zamay SS (1992) Electric field in the equatorial ionosphere. Planet Space Sci 40(7):941952. https://doi.org/10.1016/0032-0633(92)90134-A

Denisenko VV, Zamai SS, Kitaev AV (2003) The effect of viscous friction between the solar wind and plasma sheet on electric field generation in the magnetosphere. Geomagn Aeron 43(6):680-686

Denisenko VV, Biernat HK, Erkaev NV, Semenov VS (2006) Mathematical model of magnetic field perturbations by currents in the Earth's magnetosphere. In: Rucker HO, Kurth WS, Mann G (eds) Planetary radio emissions VI. Austrian Academy of Sciences Press, Vienna, pp 309-316

Denisenko VV, Biernat HK, Mezentsev AV, Shaidurov VA, Zamay SS (2008) Modification of conductivity due to acceleration of the ionospheric medium. Ann Geophys 26:2111-2130. https://doi.org/10.5194/ angeo-26-2111-2008

Denisenko VV, Bychkov VV, Pomozov EV (2009) Calculation of atmospheric electric fields penetrating from the ionosphere. Geomagn Aeron 49(8):1275-1277. https://doi.org/10.1134/S0016793209080489

Denisenko VV, Ampferer M, Pomozov EV, Kitaev AV, Hausleitner W, Stangl G, Biernat HK (2013) On electric field penetration from ground into the ionosphere. J Atmos Solar Terr Phys 102:341-353. https ://doi.org/10.1016/j.jastp.2013.05.019

Fejer BG (1981) The equatorial ionospheric electric fields. A review. J Atmos Terr Phys 43(5/6):377-386. https://doi.org/10.1016/0021-9169(81)90101-X

Feynman RP, Leighton RB, Sands M (1964) The Feynman lectures on physics, vol 2. Addison-Wesley Publishing Co Inc, London

Forbes JM (1981) The equatorial electrojet. Rev Geophys 19:469-504. https://doi.org/10.1029/RG019i003p 00469

Fullekrug M, Rycroft MJ (2006) The contribution of sprites to the global atmospheric electric circuit. Earth Planets Space 58:1193-1196. https://doi.org/10.1186/BF03352009

Gurevich AV, Krylov AL, Tsedilina EE (1976) Electric field in the Earth's magnetosphere and ionosphere. Space Sci Rev 191:59-160

Haldoupis C, Rycroft M, Williams E, Price C (2017) Is the "Earth-ionosphere capacitor" a valid component in the atmospheric global electric circuit? J Atmos Solar Terr Phys 164:27-131. https://doi. org/10.1016/j.jastp.2017.08.012

Handbook of Geophysics, United States Air Force (1960) The Macmillan Company, New York

Hargreaves JK (1979) The upper atmosphere and solar-terrestrial relations. Van Nostrand Reinold Co Ltd, NewYork

Harrison RG, Aplin KL, Rycroft MJ (2010) Atmospheric electricity coupling between earthquake regions and the ionosphere. J Atmos Sol Terr Phys 72:376-381. https://doi.org/10.1016/j.jastp.2009.12.004

Harrison RG, Aplin KL, Rycroft MJ (2014) Brief communication: earthquake-cloud coupling through the global atmospheric electric circuit. Nat Hazards Earth Syst Sci 14:773-777. https://doi.org/10.5194/ nhess-14-773-2014

Hastings DA, Dunbar PK, Elphingstone GM, Bootz M, Murakami H, Maruyama H, Masaharu H, Holland P, Payne J, Bryant NA, Logan TL, Muller J-P, Schreier G, MacDonald JS (eds) (1999) The global land one-kilometer base elevation (GLOBE) digital elevation model, version 1.0. National Oceanic and Atmospheric Administration, National Geophysical Data Center, Digital data base on the World Wide Web. http://www.ngdc.noaa.gov/mgg/topo/globe.html

Hays PB, Roble RG (1979) A quasi-static model of global atmospheric electricity. 1. The lower atmosphere. J Geophys Res 84(A7):3291-3305. https://doi.org/10.1029/JA084iA07p03291

Jansky J, Pasko VP (2014) Charge balance and ionospheric potential dynamics in time-dependent global electric circuit model. J Geophys Res Space Phys 119:10,184-10,203. https://doi.org/10.1002/2014J A020326

Jansky J, Pasko VP (2015) Effects of conductivity perturbations in time-dependent global electric circuit model. J Geophys Res 120:10654-10668. https://doi.org/10.1002/2015JA021604 
Jansky J, Lucas GM, Kalb C, Bayona V, Peterson MJ, Deierling W, Flyer N, Pasko VP (2017) Analysis of the diurnal variation of the global electric circuit obtained from different numerical models. J Geophys Res 122:12,906-12,917. https://doi.org/10.1002/2017JD026515

Kamide Y, Matsushita SJ (1979) Simulation studies of ionospheric electric fields and currents in relation to field aligned currents. 1. Queit Periods. J Geophys Res 84(8):4083-4098

Kamide Y, Richmond AD, Matsushita S (1981) Estimation of ionospheric electric fields, ionospheric currents and field-aligned currents from ground magnetic records. J Geophys Res 86(2):801-813. https://doi.org/10.1029/JA086iA02p00801

Kartalev MD, Rycroft MJ, Fuellekrug M, Papitashvili VO, Keremidarska VI (2006) A possible explanation for the dominant effect of South American thunderstorms on the Carnegie curve. J Atmos Sol Terr Phys 68:457-468. https://doi.org/10.1016/j.jastp.2005.05.012

Kelley MC (2009) The Earth's ionosphere: plasma physics and electrodynamics. Academic Press, Burlington

Kudintseva IG, Nickolaenko AP, Rycroft MJ, Odzimek A (2016) AC and DC global electric circuit properties and the height profile of atmospheric conductivity. Ann Geophys 59:A0545. https://doi. org/10.4401/ag-6870

Kuo CL, Lee LC, Huba JD (2014) An improved coupling model for the lithosphere-atmosphere-ionosphere system. J Geophys Res Space Phys 119:3189-3205. https://doi.org/10.1002/2013JA019392

Lucas GM, Baumgaertner AJG, Thayer JP (2015) A global electric circuit model within a community climate model. J Geophys Res Atmos 120:12,054-12,066. https://doi.org/10.1002/2015JD023562

Makino M, Ogawa T (1985) Quantitative estimation of global circuit. J Geophys Res 90(D4):5961-5966. https://doi.org/10.1029/JD090iD04p05961

Mareev EA (2010) Achievements and prospects of the global electric circuit. Phys Usp 53:504-534. https://doi.org/10.3367/UFNr.0180.201005h.0527

Mareev EA, Volodin EM (2014) Variation of the global electric circuit and ionospheric potential in a global circulation model. Geophys Res Lett 41:9009-9016. https://doi.org/10.1002/2014GL062352

Molchanov O, Hayakawa M (2008) Seismo-electromagnetics and related phenomena: history and latest results. TERRAPUB, Tokyo

Namgaladze AA, Zolotov OV, Prokhorov BE (2013) Numerical simulation of the variations of the ionospheric total electron content observed before the earthquake January 12, 2010 at Haiti island. Geomagn Aeron 53:522-530. https://doi.org/10.1134/S0016793213030122

Odzimek A, Lester M, Kubicki M (2010) EGATEC: a new high-resolution engineering model of the global atmospheric electric circuit-currents in the lower atmosphere. J Geophys Res 115:D18207. https://doi.org/10.1029/2009JD013341

Roble RG, Hays PB (1979) A quasi-static model of global atmospheric electricity. 2. Electric coupling between the upper and lower atmosphere. J Geophys Res 84(A12):7247-7256. https://doi. org/10.1029/JA084iA12p07247

Rycroft MJ, Harrison GR (2012) Electromagnetic atmosphere-plasma coupling: the global atmospheric electric circuit. Space Sci Rev 168:363-384. https://doi.org/10.1007/s11214-011-9830-8

Rycroft MJ, Odzimek A (2010) Effects of lightning and sprites on the ionospheric potential, and threshold effects on sprite initiation, obtained using an analog model of the global atmospheric electric circuit. J Geophys Res 115:A00E37. https://doi.org/10.1029/2009JA014758

Rycroft MJ, Israelsson S, Price C (2000) The global atmospheric electric circuit, solar activity and climate change. J Atmos Sol Terr Phys 62:1563-1576. https://doi.org/10.1016/S1364-6826(00)00112 $-7$

Rycroft MJ, Odzimek A, Arnold NF, Fullekrug M, Kulak A, Neubert T (2007) New model simulations of the global atmospheric electric circuit driven by thunderstorms and electrified shower clouds: the roles of lightning and sprites. J Atmos Sol Terr Phys 69:2485-2509. https://doi.org/10.1016/j. jastp.2007.09.004

Rycroft MJ, Harrison GR, Nicoll KA, Mareev EA (2008) An overview of Earth's global electric circuit and atmospheric conductivity. Space Sci Rev 137:83-105. https://doi.org/10.1007/s11214-008-9368-6

Rycroft MJ, Nicoll KA, Aplin KL, Harrison RG (2012) Recent advances in global electric circuit coupling between the space environment and the troposphere. J Atmos Sol Terr Phys 90-91:198-211. https://doi.org/10.1016/j.jastp.2012.03.015

Schlegel K, Fullekrug M (2002) 50 Years of Schumann resonance. Physik Unserer Zeit 33(6):256-264 [in German]

Slyunyaev NN, Mareev EA, Zhidkov AA (2015) On the variation of the ionospheric potential due to large-scale radioactivity enhancement and solar activity. J Geophys Res Space Phys 120:70607082. https://doi.org/10.1002/2015JA021039 
Spiro RW, Reiff PH, Maher LJ (1982) Precipitating electron energy flux and auroral zone conductivites—an empirical model. J Geophys Res 87(1):8215-8227. https://doi.org/10.1029/JA087iA10p08215

Tinsley BA (2008) The global atmospheric electric circuit and its effects on cloud microphysics. Rep Prog Phys 71:066801. https://doi.org/10.1088/0034-4885/71/6/066801

Tinsley BA, Zhou L (2006) Initial results of a global circuit model with variable stratospheric and tropospheric aerosols. J Geophys Res 111:D16205. https://doi.org/10.1029/2005JD006988

Tsyganenko NA, Sitnov MI (2007) Magnetospheric configurations from a high-resolution data-based magnetic field model. J Geophys Res 112:A06225. https://doi.org/10.1029/2007JA012260

Weimer DR (1999) Substorm influence on the ionospheric electric potentials and currents. J Geophys Res 104(1):185-197. https://doi.org/10.1029/1998JA900075

Williams ER (2009) The global electrical circuit: a review. Atmos Res 91:140-152. https://doi. org/10.1016/j.atmosres.2008.05.018

Williams ER, Mareev EA (2014) Recent progress on the global electrical circuit. Atmos Res 135-136:298227. https://doi.org/10.1016/j.atmosres.2013.05.015

World Geodetic System (1984) (WGS 84). http://earth-info.nga.mil/GandG/wgs84

\section{Affiliations}

\section{V. Denisenko, ${ }^{1,2}$ M. J. Rycroft ${ }^{3,4} \cdot$ R. G. Harrison ${ }^{5,6}$}

1 Institute of Computational Modelling, Siberian Branch, Russian Academy of Sciences, Krasnoyarsk, Russia 660036

2 Siberian Federal University, Krasnoyarsk, Russia 660041

3 CAESAR Consultancy, Cambridge CB3 9HW, UK

4 Centre for Space, Atmospheric and Oceanic Science, Department of Electronic and Electrical Engineering, University of Bath, Bath BA2 7AY, UK

5 Department of Meteorology, University of Reading, Earley Gate, Reading RG6 6BB, UK

6 Department of Electronic and Electrical Engineering, University of Bath, Bath BA2 7AY, UK 